\title{
A unified theory of cone metric spaces and its applications to the fixed point theory
}

\section{Petko D Proinov}

\section{"Correspondence:}

proinov@uni-plovdiv.bg

Faculty of Mathematics and

Informatics, Plovdiv University,

Plovdiv, 4000, Bulgaria

\begin{abstract}
In this paper, we develop a unified theory for cone metric spaces over a solid vector space. As an application of the new theory, we present full statements of the iterated contraction principle and the Banach contraction principle in cone metric spaces over a solid vector space. We propose a new approach to such cone metric spaces. We introduce a new notion of strict vector ordering, which is quite natural and it is easy to use in the cone metric theory and its applications to the fixed point theory. This notion plays the main role in the new theory. Among the other results in this paper, the following is perhaps of most interest. Every ordered vector space with convergence can be equipped with a strict vector ordering if and only if it is a solid vector space. Moreover, if the positive cone of an ordered vector space with convergence is solid, then there exists only one strict vector ordering on this space. Also, in this paper we present some useful properties of cone metric spaces, which allow us to establish convergence results for Picard iteration with a priori and a posteriori error estimates.

MSC: $54 \mathrm{H} 25 ; 47 \mathrm{H} 10 ; 46 \mathrm{~A} 19 ; 65 \mathrm{~J} 15 ; 06 \mathrm{~F} 30$
\end{abstract}

Keywords: cone metric space; solid vector space; fixed points; Picard iteration; iterated contraction principle; Banach contraction principle

\section{Introduction}

In 1905, the famous French mathematician Maurice Fréchet [1, 2] introduced the concept of metric spaces. In 1934, his PhD student, the Serbian mathematician, Đuro Kurepa [3], introduced more abstract metric spaces, in which the metric takes values in an ordered vector space. In the literature, the metric spaces with vector valued metric are known under various names: pseudometric spaces [3, 4], $K$-metric spaces [5-7], generalized metric spaces $[8,9]$, vector-valued metric spaces [10], cone-valued metric spaces [11, 12], cone metric spaces $[13,14]$.

It is well known that cone metric spaces and cone normed spaces have deep applications in the numerical analysis and the fixed point theory. Some applications of cone metric spaces can be seen in Collatz [4], Zabrejko [6], Rus, Petruşel, Petruşel [15] and in references therein. Schröder $[16,17]$ was the first who pointed out the important role of cone metric spaces in the numerical analysis. The famous Russian mathematician Kantorovich [18] was the first who showed the importance of cone normed spaces for the numerical analysis.

Starting from 2007, many authors have studied cone metric spaces over solid Banach spaces and fixed point theorems in such spaces (Huang and Zhang [13], Rezapour and 
Hamlbarani [19], Wardowski [20], Pathak and Shahzad [21], Şahin and Telsi [22], AminiHarandi and Fakhar [23], Sönmez [24], Latif and Shaddad [25], Turkoglu and Abuloha [26], Khamsi [27], Radenović and Kadelburg [28], Khani and Pourmahdian [29], Asadi, Vaezpour and Soleimani [30], Ding, Kadelburg, Karapinar, Radenović [31], Lin, Chen, Jovanović, Wu [32] and others).

Recently, some authors have studied cone metric spaces over solid topological vector spaces and fixed point theorems in such spaces (Beg, Azam and Arshad [33], Du [34, 35], Azam, Beg and Arshad [36], Janković, Kadelburg and Radenović [14], Kadelburg, Radenović and Rakočević [37], Arandelović and Kečkić [38], Simić [39], Çakalli, Sönmez and Genç [40], Asadi, Rhoades and Soleimani [41] and others).

The purpose of this paper is three-fold. First, we develop a unified theory for solid vector spaces. Second, we develop a unified theory for cone metric spaces over a solid vector space. Third, we present full statements of the iterated contraction principle and the Banach contraction principle in cone metric spaces over a solid vector space. The main results of the paper generalize, extend and complement some recent results of $\mathrm{Du}$ [34], Kadelburg, Radenović and Rakočević [37], Pathak and Shahzad [21], Wardowski [20], Radenović and Kadelburg [28] and others.

The paper is structured as follows.

In Section 2, we introduce an axiomatic definition of a vector space with convergence, which does not require neither axiom for the uniqueness of the limit of a convergent sequence nor axiom for convergence of subsequences of a convergent sequence (Definition 2.1). Our axioms are enough to prove some fixed point theorems in cone metric spaces over solid vector spaces.

In Section 3, we establish a necessary and sufficient condition for the interior of a solid cone in a vector space with convergence (Theorem 3.1).

In Section 4, we introduce the definition of an ordered vector space with convergence and the well-known theorem that the vector orderings and cones in a vector space with convergence are in one-to-one correspondence.

In Section 5, we introduce an axiomatic definition of the new notion of a strict vector ordering on an ordered vector space with convergence (Definition 5.1). Then we show that an ordered vector space with convergence can be equipped with a strict vector ordering $\prec$ if and only if it is a solid vector space (Theorem 5.1). Moreover, if the positive cone of an ordered vector space with convergence is solid, then there exists only one strict vector ordering on this space. Hence, the strict vector orderings and solid cones in an vector space with convergence are in one-to-one correspondence. It turns out that a solid vector space can be defined as an ordered vector space with convergence equipped with a strict vector ordering (Corollary 5.1).

In Section 6, we show that every solid vector space can be endowed with an order topology $\tau$ and that $x_{n} \rightarrow x$ implies $x_{n} \stackrel{\tau}{\rightarrow} x$ (Theorems 6.1 and 6.2). As a consequence, we show that every convergent sequence in a solid vector space is bounded and has a unique limit (Theorem 6.3).

In Section 7, using the Minkowski functional, we show that the order topology on every solid vector space is normable with a monotone norm (Theorem 7.1). We also show that every normal solid vector space $Y$ is normable in the sense that there exists a norm $\|\cdot\|$ on $Y$ such that $x_{n} \rightarrow x$ if and only if $x_{n} \stackrel{\|\cdot\|}{\rightarrow} x$ (Theorem 7.3). At the end of the section, we show that the convergence of sequences in a normal solid vector space has the properties of the 
convergence in $\mathbb{R}$ (Theorem 7.4). This result shows that the sandwich theorem plays an important role in solid vector spaces. In particular, Theorem 7.4 shows that a sequence $\left(x_{n}\right)$ in a normal solid vector space converges to a vector $x$ if and only if for every vector $c \succ 0$, we have $x-c \prec x_{n} \prec x+c$ for sufficiently large $n$.

In Section 8, we introduce the classical definitions of cone metric spaces and cone normed spaces. Note that in our definition of a cone normed space $(X,\|\cdot\|)$ we allow $X$ to be a vector space over an arbitrary normed field $\mathbb{K}$.

In Section 9, we study cone metric spaces over solid vector spaces. The theory of such cone metric spaces is very close to the theory of the usual metric spaces. For example, every cone metric space over a solid vector space is a metrizable topological space (Theorem 9.2) and in such spaces the nested ball theorem holds (Theorem 9.10). Moreover, the cone metric is equivalent to a metric which preserve some inequalities. Among the other results in this section, we prove that every cone normed space over a solid vector space is normable (Theorem 9.4). Also, in this section, we give some useful properties of cone metric spaces which allow us to establish convergence results for Picard iteration with a priori and a posteriori error estimates (Theorem 9.6 and Theorem 9.9). Some of the results in this section generalize, extend and complement some results of Du [34], Kadelburg, Radenović and Rakočević [37, 42], Çakalli, Sönmez and Genç [40], Simić [39], Abdeljawad and Rezapour [43], Arandelović and Kečkić [38], Amini-Harandi and Fakhar, [23], Khani and Pourmahdian [29], Sönmez [24], Asadi, Vaezpour and Soleimani [30], Şahin and Telsi [22] and Azam, Beg and Arshad [36].

In Section 10, we establish a full statement of the iterated contraction principle in cone metric spaces over a solid vector space. The main result of this section (Theorem 10.1) generalizes, extends and complements some results of Pathak and Shahzad [21], Wardowski [20], Ortega and Rheinboldt [44, Theorem 12.3.2] and others.

In Section 11, we establish a full statement of the Banach contraction principle in cone metric spaces over a solid vector space. The main result of this section (Theorem 11.1) generalizes, extends and complements some results of Rezapour and Hamlbarani [19], Du [34], Radenović and Kadelburg [28] and others.

\section{Vector spaces with convergence}

In this section, we introduce a kind of sequential convergence in a vector space. There is a classical axiomatization of sequential convergence on an arbitrary set (see Fréchet [2], Dudley [45], Frič [46], Rus [47] Petruşel and Rus [48], Gutierres and Hofmann [49], Rus, Petruşel and Petruşel [15] and references therein). Let $Y$ be any nonempty set and $S$ be the set of all infinite sequences in $Y$. Let us recall that a sequential convergence or $\mathcal{L}$-convergence on $Y$ is a relation $\rightarrow$ between $S$ and $Y$ such that:

(A0) If $x_{n} \rightarrow x$ and $x_{n} \rightarrow y$, then $x=y$.

(A1) If $x_{n}=x$ for all $n$, then $x_{n} \rightarrow x$.

(A2) If $x_{n} \rightarrow x$ and $\left(y_{n}\right)$ is any subsequence of $\left(x_{n}\right)$, then $y_{n} \rightarrow x$.

A set endowed with a sequential convergence is called a sequential space or $\mathcal{L}$-space.

Some authors consider the sequential convergence in vector spaces (see Jakubík [50], Schröder [16, 17], Collatz [4], De Pascale, Marino and Pietramala [51] , Zabrejko [6], Rus, Petruşel and Petruşel [15] and references therein). In the paper [50] of Jakubík are given the following axioms for sequential convergence in a vector space $Y$ : 
(B1) If $x_{n} \rightarrow x$ and $y_{n} \rightarrow y$, then $x_{n}+y_{n} \rightarrow x+y$.

(B2) If $x_{n} \rightarrow x$ and $\lambda \in \mathbb{R}$, then $\lambda x_{n} \rightarrow \lambda x$.

(B3) If $\lambda_{n} \rightarrow \lambda$ in $\mathbb{R}$ and $x \in Y$, then $\lambda_{n} x \rightarrow \lambda x$.

(B4) If $x_{n} \rightarrow x$ and $\left(y_{n}\right)$ is any subsequence of $\left(x_{n}\right)$, then $y_{n} \rightarrow x$.

(B5) If $x_{n} \rightarrow x$ and $x_{n} \rightarrow y$, then $x=y$.

It is clear that a vector space $Y$ satisfying axioms (B1)-(B5) is a sequential space.

In this study, we do not need axioms (B4) and (B5). It is possible to prove some fixed point theorems without using axioms (B4) and (B5). We introduce the following definition of weak sequential convergence in a vector space $Y$.

Definition 2.1 Let $Y$ be a real vector space and $S$ be the set of all infinite sequences in $Y$. A binary relation $\rightarrow$ between $S$ and $Y$ is called a convergence on $Y$ if it satisfies the following axioms:

(C1) If $x_{n} \rightarrow x$ and $y_{n} \rightarrow y$, then $x_{n}+y_{n} \rightarrow x+y$.

(C2) If $x_{n} \rightarrow x$ and $\lambda \in \mathbb{R}$, then $\lambda x_{n} \rightarrow \lambda x$.

(C3) If $\lambda_{n} \rightarrow \lambda$ in $\mathbb{R}$ and $x \in Y$, then $\lambda_{n} x \rightarrow \lambda x$.

The pair $(Y, \rightarrow)$ is said to be a vector space with convergence. If $x_{n} \rightarrow x$, then $\left(x_{n}\right)$ is said to be a convergent sequence in $Y$, and the vector $x$ is said to be a limit of $\left(x_{n}\right)$.

The following two properties of the convergence in a vector space $Y$ follow immediately from Definition 2.1.

(C4) If $x_{n}=x$ for all $n$, then $x_{n} \rightarrow x$.

(C5) The convergence and the limits of a sequence do not depend on the change of finitely many of its terms.

Definition 2.2 Let $(Y, \rightarrow)$ be a vector space with convergence.

(a) A set $A \subset Y$ is said to be (sequentially) open if $x_{n} \rightarrow x$ and $x \in A$ imply $x_{n} \in A$ for all but finitely many $n$.

(b) A set $A \subset Y$ is said to be (sequentially) closed if $x_{n} \rightarrow x$ and $x_{n} \in A$ for all $n$ imply $x \in A$.

Remark 2.1 Let $(Y, \rightarrow)$ be a vector space with convergence. It is easy to prove that if a set $A \subset Y$ is open, then $Y \backslash A$ is closed. Let us note that the converse holds true provided that every subsequence of a convergent sequence in $Y$ is convergent with the same limits.

The following lemma follows immediately from the first part of Definition 2.2.

Lemma 2.1 Let $(Y, \rightarrow)$ be a vector space with convergence. The open sets in $Y$ satisfies the following properties:

(i) $\varnothing$ and $Y$ are open.

(ii) The union of any family of open sets is open.

(iii) The intersection of any finite family of open sets is open.

Remark 2.2 Lemma 2.1 shows that the family of all open subsets of $(Y, \rightarrow)$ defines a topology on $Y$. Note that in this paper we will never consider this topology on $Y$.

Lemma 2.2 Let $(Y, \rightarrow)$ be a vector space with convergence. Suppose $U$ and $V$ are two nonempty subsets of $Y$. Then the following statements hold true: 
(i) If $U$ is open and $\lambda>0$, then $\lambda U$ is open.

(ii) If $U$ or $V$ is open, then $U+V$ is open.

Proof (i) Let $\lambda>0$ and $U$ be an open subset of $Y$. Suppose $\left(x_{n}\right)$ is a convergent sequence in $Y$ with a limit $x \in \lambda U$. Then there exists a vector $a \in U$ such that $x=\lambda a$. Consider the sequence $\left(a_{n}\right)$ defined by $a_{n}=\frac{1}{\lambda} x_{n}$. It follows from (C2) that $a_{n} \rightarrow a$ since $a=\frac{1}{\lambda} x$. Taking into account that $U$ is open and $a \in U$, we conclude that $a_{n} \in U$ for all but finitely many $n$. Then $x_{n} \in \lambda U$ for the same $n$ since $x_{n}=\lambda a_{n}$. Therefore, the set $\lambda U$ is open.

(ii) Let $U$ be an arbitrary subset of $Y$ and $V$ be an open subset of $Y$. Suppose $\left(x_{n}\right)$ is a convergent sequence in $Y$ with limit $x \in U+V$. Then there exist $a \in U$ and $b \in V$ such that $x=a+b$. Consider the sequence $\left(b_{n}\right)$ defined by $b_{n}=x_{n}-a$. It follows from (C1) and (C4) that $b_{n} \rightarrow b$ since $b=x-a$. Taking into account that $V$ is open and $b \in V$, we conclude that $b_{n} \in V$ for all but finitely many $n$. Then $x_{n} \in U+V$ for these $n$ since $x_{n}=a+b_{n}$. Therefore, the set $U+V$ is open.

Due to the first two parts of Lemma 2.1, we can give the following definition.

Definition 2.3 Let $A$ be a subset of a vector space with convergence. The interior $A^{\circ}$ of $A$ is called the biggest open subset contained in $A$, that is, $A^{\circ}=\bigcup U$ where $\bigcup$ ranges through the family of all open subsets of $Y$ contained in $A$.

The following lemma follows immediately from Definition 2.3.

Lemma 2.3 Let $A$ and $B$ be two subsets of a vector space with convergence. Then

$$
A \subset B \text { implies } A^{\circ} \subset B^{\circ} \text {. }
$$

Example 2.1 Let $(Y, \tau)$ be an arbitrary topological vector space and let $\stackrel{\tau}{\rightarrow}$ be the $\tau$ convergence in $Y$. Obviously, $(Y, \stackrel{\tau}{\rightarrow})$ is a vector space with convergence. It is well known that every $\tau$-open subset of $(Y, \tau)$ is sequentially open and every $\tau$-closed set is sequentially closed. Recall also that a topological space is called a sequential topological space if it satisfies one of the following equivalent conditions:

(a) Every sequentially open subset of $Y$ is $\tau$-open.

(b) Every sequentially closed subset of $Y$ is $\tau$-closed.

According to a well-known theorem of Franklin [52], every first countable topological vector space is a sequential space. For more details on sequential topological spaces, we refer the reader to the survey paper of Goreham [53].

\section{Solid cones in vector spaces with convergence}

In this section, we establish a useful criterion for the interior of a solid cone. This criterion will play an important role in Section 5.

For more on cone theory, see the classical survey paper of Krein and Rutman [54], the classical monographs of Krasnoselskii [55, Chapter 1], Deimling [56, Chapter 6], Zeidler [57, Section 1.6] as well as the recent monograph of Aliprantis and Tourky [58].

Definition 3.1 Let $(Y, \rightarrow)$ be a vector space with convergence. A nonempty closed subset $K$ of a vector space $Y$ is called a cone if it satisfies the following properties: 
(i) $\lambda K \subset K$ for any $\lambda \geq 0$;

(ii) $K+K \subset K$;

(iii) $K \cap(-K)=\{0\}$.

A cone $K$ is called trivial if $K=\{0\}$. A nontrivial cone $K$ is said to be a solid cone if its interior is nonempty.

Lemma 3.1 Let $(Y, \rightarrow)$ be a vector space with convergence and $K$ be a cone in $Y$. Then there is at most one nonempty open subset $U$ of $K$ satisfying the following conditions:

(i) $\lambda U \subset U$ for any $\lambda>0$;

(ii) $K+U \subset U$;

(iii) $0 \notin U$.

Proof Let $U$ be a nonempty open subsets of $K$ satisfying conditions (i)-(iii). First we shall prove that every nonempty open subset $V$ of $K$ is a subset of $U$. Let a vector $x \in V$ be fixed. Choose a vector $a \in U$ with $a \neq 0$. This is possible since $U$ is nonempty and $0 \notin U$. Consider the sequence $\left(x_{n}\right)$ in $Y$ defined by $x_{n}=x-\frac{1}{n} a$. It follows from (C1) and (C4) that $x_{n} \rightarrow x$. Since $V$ is open and $x \in V$, then there exists $n \in \mathbb{N}$ such that $x_{n} \in V$. Therefore, $x_{n} \in K$ since $V \subset K$. On the other hand it follows from (i) that $\frac{1}{n} a \in U$ since $a \in U$. Then from (ii) we conclude that $x=x_{n}+\frac{1}{n} a \in U$ which proves that $V \subset U$. Now if $U$ and $V$ are two nonempty open subsets of $K$ satisfying conditions (i)-(iii), then we have both $V \subset U$ and $U \subset V$ which means that $U=V$.

Now we are ready to establish a criterion for the interior of a solid cone.

Theorem 3.1 Let $(Y, \rightarrow)$ be a vector space with convergence and $K$ be a cone in $Y$. Then the interior $K^{\circ}$ of $K$ has the following properties:

(i) $\lambda K^{\circ} \subset K^{\circ}$ for any $\lambda>0$;

(ii) $K+K^{\circ} \subset K^{\circ}$;

(iii) $0 \notin K^{\circ}$.

Conversely, if a nonempty open subset $K^{\circ}$ of $K$ satisfies properties (i)-(iii), then $K^{\circ}$ is just the interior of $K$.

Proof First part. We shall prove that the interior $K^{\circ}$ of a solid cone $K$ satisfies properties (i)-(iii).

(i) Let $\lambda>0$. It follows from Lemma 2.2(i) that $\lambda K^{\circ}$ is open. From $K^{\circ} \subset K$ and $\lambda K \subset K$, we conclude that $\lambda K^{\circ} \subset K$. This inclusion and Lemma 2.3 imply $\lambda K^{\circ} \subset K^{\circ}$.

(ii) According to Lemma 2.2(ii) $K+K^{\circ}$ is an open set. It follows from $K^{\circ} \subset K$ and $K+K \subset K$ that $K+K^{\circ} \subset K$. Now from Lemma 2.3, we obtain that $K+K^{\circ} \subset K^{\circ}$.

(iii) Assume that $0 \in K^{\circ}$. Since $K$ is nonempty and nontrivial, then we can choose a vector $a \in K$ with $a \neq 0$. By axiom (C3), $-\frac{1}{n} a \rightarrow 0$. Taking into account that $K^{\circ}$ is open, we conclude that there exists $n \in \mathbb{N}$ such that $-\frac{1}{n} a \in K^{\circ}$. Then it follows from (i) that $-a \in K^{\circ}$. Since $K^{\circ} \subset K$, we have both $a \in K$ and $-a \in K$, which implies $a=0$. This is a contradiction which proves that $0 \notin K^{\circ}$.

Second part. The second part of the theorem follows from Lemma 3.1. Indeed, suppose that $K^{\circ}$ is a nonempty open subset of $K$ satisfying properties (i)-(iii). Then by Lemma 3.1, we conclude that $K^{\circ}$ is a unique nonempty open subset of $K$ satisfying these properties. 
On the other hand, it follows from the first part of the theorem that the interior of $K$ also satisfies properties (i)-(iii). Therefore, $K^{\circ}$ coincide with the interior of $K$.

\section{Ordered vector spaces with convergence}

Recall that a binary relation $\preceq$ on a set $Y$ is said to be an ordering on $Y$ if it is reflexive, antisymmetric and transitive.

Definition 4.1 Let $(Y, \rightarrow)$ be a vector space with convergence. An ordering $\preceq$ on $Y$ is said to be a vector ordering if it is compatible with the algebraic and convergence structures on $Y$ in the sense that the following are true:

(V1) If $x \preceq y$, then $x+z \preceq y+z$;

(V2) If $\lambda \geq 0$ and $x \preceq y$, then $\lambda x \preceq \lambda y$;

(V3) If $x_{n} \rightarrow x, y_{n} \rightarrow y, x_{n} \preceq y_{n}$ for all $n$, then $x \preceq y$.

A vector space with convergence $(Y, \rightarrow)$ equipped with a vector ordering $\preceq$ is called an ordered vector space with convergence and is denoted by $(Y, \preceq, \rightarrow)$. If the convergence $\rightarrow$ on $Y$ is produced by a vector topology $\tau$, we sometimes write $(Y, \tau, \preceq)$ instead of $(Y, \preceq, \rightarrow)$. Analogously, if the convergence $\rightarrow$ on $Y$ is produced by a norm $\|\cdot\|$, we sometimes write $(Y,\|\cdot\|, \preceq)$.

Axiom (V3) is known as passage to the limit in inequalities. Obviously, it is equivalent to the following statement:

$\left(\mathrm{V}^{\prime}\right)$ If $x_{n} \rightarrow x, x_{n} \succeq 0$ for all $n$, then $x \succeq 0$.

Every vector ordering $\preceq$ on an ordered vector space $(Y, \preceq, \rightarrow)$ satisfies also the following properties:

(V4) If $\lambda \leq 0$ and $x \preceq y$, then $\lambda x \succeq \lambda y$;

(V5) If $\lambda \leq \mu$ and $x \succeq 0$, then $\lambda x \preceq \mu x$;

(V6) If $\lambda \leq \mu$ and $x \preceq 0$, then $\lambda x \succeq \mu x$;

(V7) If $x \preceq y$ and $u \preceq v$, then $x+u \preceq y+v$.

Definition 4.2 Let $(Y, \preceq, \rightarrow)$ be an ordered vector space with convergence. The set

$$
Y_{+}=\{x \in Y: x \succeq 0\}
$$

is called the positive cone of the ordering $\preceq$ or positive cone of $Y$.

The following well-known theorem shows that the positive cone is indeed a cone. It shows also that the vector orderings and cones in a vector space with convergence are in one-to-one correspondence.

Theorem 4.1 Let $(Y, \rightarrow)$ be a vector space with convergence. If a relation $\preceq$ is a vector ordering on $Y$, then its positive cone is a cone in $Y$. Conversely, if a subset $K$ of $Y$ is a cone, then the relation $\preceq$ on $Y$ defined by means of

$$
x \preceq y \text { if and only if } y-x \in K
$$

is a vector ordering on $Y$ whose positive cone coincides with $K$. 
Definition 4.3 Let $(Y, \preceq, \rightarrow)$ be an ordered vector space with convergence.

(a) A set $A \subset Y$ is called bounded if there exist two vectors in $a, b \in Y$ such that $a \preceq x \preceq b$ for all $x \in A$.

(b) A sequence $\left(x_{n}\right)$ in $Y$ is called bounded if the set of its terms is bounded.

(c) A sequence $\left(x_{n}\right)$ in $Y$ is called increasing if $x_{1} \preceq x_{2} \preceq \cdots$.

(d) A sequence $\left(x_{n}\right)$ in $Y$ is called decreasing if $x_{1} \succeq x_{2} \succeq \cdots$.

Definition 4.4 An ordered vector space with convergence $(Y, \preceq, \rightarrow)$ is called a solid vector space if its positive cone is solid.

Definition 4.5 An ordered vector space with convergence $(Y, \preceq, \rightarrow)$ is called a normal vector space whenever for arbitrary sequences $\left(x_{n}\right),\left(y_{n}\right),\left(z_{n}\right)$ in $Y$,

$$
x_{n} \preceq y_{n} \preceq z_{n} \quad \text { for all } n \text { and } x_{n} \rightarrow x \text { and } z_{n} \rightarrow x \text { imply } \quad y_{n} \rightarrow x \text {. }
$$

The statement (4.3) is known as sandwich theorem or rule of intermediate sequence.

Definition 4.6 An ordered vector space with convergence $(Y, \preceq, \rightarrow)$ is called a regular vector space if it satisfies one of the following equivalent conditions.

(a) Every bounded increasing sequence in $Y$ is convergent.

(b) Every bounded decreasing sequence in $Y$ is convergent.

\section{Strict vector orderings and solid cones}

In this section, we introduce the new notion of strict vector ordering and prove that an ordered vector space with convergence can be equipped with a strict vector ordering if and only if it is a solid vector space.

Recall that a nonempty binary relation $\prec$ on a set $Y$ is said to be a strict ordering on $Y$ if it is irreflexive, asymmetric and transitive.

Definition 5.1 Let $(Y, \preceq, \rightarrow)$ be an ordered vector space with convergence. A strict ordering $\prec$ on $Y$ is said to be a strict vector ordering if it is compatible with the vector ordering, the algebraic structure and the convergence structure on $Y$ in the sense that the following are true:

(S1) If $x \prec y$, then $x \preceq y$;

(S2) If $x \preceq y$ and $y \prec z$, then $x \prec z$;

(S3) If $x \prec y$, then $x+z \prec y+z$;

(S4) If $\lambda>0$ and $x \prec y$, then $\lambda x \prec \lambda y$;

(S5) If $x_{n} \rightarrow x, y_{n} \rightarrow y$ and $x \prec y$, then $x_{n} \prec y_{n}$ for all but finitely many $n$.

An ordered vector space with convergence $(Y, \preceq, \rightarrow)$ equipped with a strict vector ordering $\prec$ is denoted by $(Y, \preceq, \prec, \rightarrow)$. It turns out that ordered vector spaces with strict vector ordering are just solid vector spaces (see Corollary 5.1 below).

Axiom (S5) is known as converse property of passage to the limit in inequalities. It is equivalent to the following statement:

$\left(\mathrm{S}^{\prime}\right)$ If $x_{n} \rightarrow 0$ and $c \succ 0$, then $x_{n} \prec c$ for all but finitely many $n$. 
Strict vector ordering $\prec$ on a ordered vector space with convergence $(Y, \preceq, \rightarrow)$ satisfies also the following properties:

(S6) If $\lambda<0$ and $x \prec y$, then $\lambda x \succ \lambda y$;

(S7) If $\lambda<\mu$ and $x>0$, then $\lambda x \prec \mu x$;

(S8) If $\lambda<\mu$ and $x \prec 0$, then $\lambda x \succ \mu x$;

(S9) If $x \prec y$ and $y \preceq z$, then $x \prec z$;

(S10) If $x \preceq y$ and $u \prec v$, then $x+u \prec y+v$;

(S11) If $x \prec c$ for each $c \succ 0$, then $x \preceq 0$;

(S12) For every finite set $A \subset Y$ consisting of strictly positive vectors, there exists a vector $c \succ 0$ such that $c \prec x$ for all $x \in A$. Moreover, for every vector $b \succ 0, c$ always can be chosen in the form $c=\lambda b$ for some $\lambda>0$;

(S13) For every finite set $A \subset Y$, there is a vector $c \succ 0$ such that $-c \prec x \prec c$ for all $x \in A$. Moreover, for every vector $b \succ 0, c$ always can be chosen in the form $c=\lambda b$ for some $\lambda>0$;

(S14) For every $x \in Y$ and every $b \in Y$ with $b \succ 0$, there exists $\lambda>0$ such that $-\lambda b \prec x \prec \lambda b$.

The proofs of properties (S6)-(S10) are trivial. Property (S14) is a special case of (S13). So we shall prove (S11)-(S13).

Proof of (S11) Let $x$ be a vector in $Y$ such that $x \prec c$ for each $c \succ 0$. Choose a vector $b \in Y$ with $b \succ 0$. It follows from (S4) that $\frac{1}{n} b \succ 0$ for each $n \in \mathbb{N}$. Hence, $x \prec \frac{1}{n} b$ for each $n \in \mathbb{N}$. Passing to the limit in this inequality, we obtain $x \preceq 0$.

Proof of (S12) Let $x$ be an arbitrary vector from $A$. Choose a vector $b \in Y$ with $b \succ 0$. Since $\frac{1}{n} b \rightarrow 0$ and $0 \prec x$, then from (S5) we deduce that $\frac{1}{n} b \prec x$ for all but finitely many $n$. Taking into account that $A$ is a finite set, we conclude that for sufficiently large $n$ we have $\frac{1}{n} b \prec x$ for all $x \in A$. Now every vector $c=\frac{1}{n} b$ with sufficiently large $n$ satisfies $c \prec x$ for all $x \in A$. To complete the proof put $\lambda=\frac{1}{n}$.

Proof of (S13) Let $x$ be an arbitrary vector from $A$. Choose a vector $b \in Y$ with $b \succ 0$. Since $\frac{1}{n} x \rightarrow 0$ and $-\frac{1}{n} x \rightarrow 0$, then from (S5) we obtain that $\frac{1}{n} x \prec b$ and $-\frac{1}{n} x \prec b$ for all but finitely many $n$. From these inequalities, we conclude that $-n b \prec x \prec n b$. Taking into account that $A$ is a finite set, we get that every vector $c=n b$ with sufficiently large $n$ satisfies $-c \prec x \prec c$ for all $x \in A$. To complete the proof, put $\lambda=n$.

The next theorem shows that an ordered vector space with convergence can be equipped with a strict vector ordering if and only if it is a solid vector space. Moreover, on every ordered vector space, there is at most one strict vector ordering. In other words, the solid cones and strict vector orderings on a vector space with convergence are in one-to-one correspondence.

Theorem 5.1 Let $(Y, \preceq, \rightarrow)$ be an ordered vector space with convergence, and let $K$ be its positive cone, i.e., $K=\{x \in Y: x \geq 0\}$. If a relation $\prec$ is a strict vector ordering on $Y$, then $K$ is a solid cone with the interior

$$
K^{\circ}=\{x \in Y: x \succ 0\} .
$$


Conversely, if $K$ is a solid cone with the interior $K^{\circ}$, then the relation $\prec$ on $Y$ defined by means of

$$
x \prec y \quad \text { if and only if } y-x \in K^{\circ}
$$

is a unique strict vector ordering on $Y$.

Proof First part. Suppose a relation $\prec$ is a strict vector ordering on $Y$. We shall prove that the set $K^{\circ}$ defined by (5.1) is a nonempty open subset of $K$ which satisfies conditions (i)-(iii) of Theorem 3.1. Then it follows from the second part of Theorem 3.1 that $K^{\circ}$ is the interior of $K$ and that $K$ is a solid cone. By the definition of strict ordering, it follows that the relation $\prec$ is nonempty. Therefore, there are at least two vectors $a$ and $b$ in $Y$ such that $a \prec b$. From (S3), we obtain $b-a \succ 0$. Therefore, $b-a \in K^{\circ}$, which proves that $K^{\circ}$ is nonempty. Now let $x_{n} \rightarrow x$ and $x \in K^{\circ}$. By the definition of $K^{\circ}$, we get $x \succ 0$. Then by (S5), we conclude that $x_{n} \succ 0$ for all but finitely many $n$ which means that $K^{\circ}$ is open. Conditions (i) and (ii) of Theorem 3.1 follow immediately from (S4) and (S10), respectively. It remains to prove that $0 \notin K^{\circ}$. Assume the contrary, that is $0 \in K^{\circ}$. By the definition of $K^{\circ}$, we get $0 \succ 0$, which is a contradiction since the relation $\prec$ is irreflexive.

Second part. Let $K$ be a solid cone and $K^{\circ}$ be its interior. Note that according to the first part of Theorem 3.1, $K^{\circ}$ has the following properties: $\lambda K^{\circ} \subset K^{\circ}$ for any $\lambda>0, K+K^{\circ} \subset K^{\circ}$ and $0 \notin K^{\circ}$. We have to prove that the relation $\prec$ defined by (5.2) is a strict vector ordering. First we shall show that $\prec$ is nonempty and irreflexive. Since $K$ is solid, $K^{\circ}$ is nonempty and nontrivial. Hence, there exists a vector $c \in K^{\circ}$ such that $c \neq 0$. Now by the definition of $\prec$, we get $0 \prec c$ which means that $\prec$ is nonempty. To prove that $\prec$ is irreflexive assume the contrary. Then there exists a vector $x \in Y$ such that $x \prec x$. Hence, $0=x-x \in K^{\circ}$ which is a contradiction since $0 \notin K^{\circ}$. Now we shall show that $\prec$ satisfies properties (S1)-(S5).

(S1) Let $x \prec y$. Using the definition (5.2), the inclusion $K^{\circ} \subset K$, and the definition of the positive cone $K$, we have

$$
x \prec y \rightarrow y-x \in K^{\circ} \rightarrow y-x \in K \rightarrow y-x \succeq 0 \rightarrow x \preceq y .
$$

(S2) Let $x \preceq y$ and $y \prec z$. Using the definition of the positive cone $K$, the definition (5.2) and the inclusion $K+K^{\circ} \subset K^{\circ}$, we get

$$
x \preceq y \text { and } y \prec z \rightarrow y-x \in K \text { and } z-y \in K^{\circ} \rightarrow z-x \in K^{\circ} \rightarrow x \prec z \text {. }
$$

(S3) follows immediately from the definition (5.2).

(S4) Let $x \preceq y$ and $\lambda>0$. Using the definition (5.2) and taking into account that $\lambda K^{\circ} \subset K^{\circ}$, we obtain

$$
x \prec y \rightarrow y-x \in K^{\circ} \rightarrow \lambda(y-x) \in K^{\circ} \rightarrow \lambda y-\lambda x \in K^{\circ} \rightarrow \lambda x \prec \lambda y .
$$

(S5) Let $x_{n} \rightarrow x, y_{n} \rightarrow y$ and $x \prec y$. This yields $y_{n}-x_{n} \rightarrow y-x$ and $y-x \in K^{\circ}$. Since $K^{\circ}$ is open, we conclude that $y_{n}-x_{n} \in K^{\circ}$ for all but finitely many $n$. Hence, $x_{n} \prec y_{n}$ for all but finitely many $n$. 
Uniqueness. Now we shall prove the uniqueness of the strict vector ordering on $Y$. Assume that $\prec$ and $<$ are two vector orderings on $Y$. It follows from the first part of the theorem that

$$
K^{\circ}=\{x \in Y: x \succ 0\}=\{x \in Y: x>0\}
$$

From this and (S3), we get for all $x, y \in Y$,

$$
x \prec y \quad \Leftrightarrow y-x>0 \quad \Leftrightarrow \quad y-x \in K^{\circ} \quad \Leftrightarrow \quad y-x>0 \quad \Leftrightarrow \quad x<y
$$

which means that relations $\prec$ and $<$ are equal.

Note that property (S13) shows that every finite set in a solid vector space is bounded. Property (S12) shows that every finite set consisting of strictly positive vectors in a solid vector space is bounded below by a positive vector.

The following assertion is an immediate consequence of Theorem 5.1. It shows that a solid vector space can be defined as an ordered vector space with convergence equipped with a strict vector ordering.

Corollary 5.1 Let $(Y, \preceq, \rightarrow)$ be an ordered vector space with convergence. Then the following statements are equivalent:

(i) $Y$ is a solid vector space.

(ii) $Y$ can be equipped with a strict vector ordering.

Remark 5.1 The strict ordering $\prec$ defined by (5.2) was first introduced in 1948 by Krein and Rutman $[54, \mathrm{p} .8]$ in the case when $K$ is a solid cone in a Banach space $Y$. In this case, they proved that $\prec$ satisfies axioms (S1)-(S4).

In conclusion of this section, we present three examples of solid vector spaces We end the section with a remark which shows that axiom (S5) plays an important role in the definition of strict vector ordering.

Example 5.1 Let $Y=\mathbb{R}^{n}$ equipped with the coordinate-wise convergence $\rightarrow$, and with coordinate-wise ordering defined by

$$
\begin{aligned}
& x \preceq y \quad \text { if and only if } \quad x_{i} \leq y_{i} \quad \text { for each } i=1, \ldots, n, \\
& x \prec y \quad \text { if and only if } \quad x_{i}<y_{i} \quad \text { for each } i=1, \ldots, n .
\end{aligned}
$$

Then $(Y, \preceq, \prec, \rightarrow)$ is a solid vector space. This space is normal and regular.

Example 5.2 Let $Y=C[0,1]$ with the max-norm $\|\cdot\|_{\infty}$. Define the pointwise ordering $\preceq$ and $\prec$ on $Y$ by means of

$$
\begin{aligned}
& x \preceq y \quad \text { if and only if } \quad x(t) \leq y(t) \quad \text { for each } t \in[0,1] \\
& x \prec y \quad \text { if and only if } \quad x(t)<y(t) \quad \text { for each } t \in[0,1] .
\end{aligned}
$$


Then $\left(Y,\|\cdot\|_{\infty}, \preceq, \prec\right)$ is a solid Banach space. This space is normal but nonregular. Consider, for example, the sequence $\left(x_{n}\right)$ in $Y$ defined by $x_{n}(t)=t^{n}$. We have $x_{1} \succeq x_{2} \succeq \cdots \succeq 0$ but $\left(x_{n}\right)$ is not convergent in $Y$.

Example 5.3 Let $Y=C^{1}[0,1]$ with the norm $\|x\|=\|x\|_{\infty}+\left\|x^{\prime}\right\|_{\infty}$. Define the ordering $\preceq$ and $\prec$ as in Example 5.2. Then $(Y,\|\cdot\|, \preceq, \prec)$ is a solid Banach space. The space $Y$ is not normal. Consider, for example, the sequences $\left(x_{n}\right)$ and $\left(y_{n}\right)$ in $Y$ defined by $x_{n}(t)=\frac{t^{n}}{n}$ and $y_{n}(t)=\frac{1}{n}$. It is easy to see that $0 \preceq x_{n} \preceq y_{n}$ for all $n, y_{n} \rightarrow 0$ and $x_{n} \not \supset 0$.

Remark 5.2 Let $(Y, \preceq, \rightarrow)$ be an arbitrary ordered vector space with convergence. Then the relation $\prec$ on $Y$ defined by

$$
x \prec y \quad \text { if and only if } \quad x \preceq y \text { and } x \neq y
$$

is a strict ordering on $Y$ and it always satisfies axioms (S1)-(S4) and properties (S5)-(S10). However, it is not in general a strict vector ordering on $Y$. For example, from the uniqueness of strict vector ordering (Theorem 5.1), it follows that $\prec$ defined by (5.3) is not a strict vector ordering in the ordered vector spaces defined in Examples 5.1-5.3.

\section{Order topology on solid vector spaces}

In this section, we show that every solid vector space can be endowed with an order topology $\tau$ and that $x_{n} \rightarrow x$ implies $x_{n} \stackrel{\tau}{\rightarrow} x$. As a consequence, we show that every convergent sequence in a solid vector space has a unique limit.

Definition 6.1 Let $(Y, \preceq, \prec, \rightarrow)$ be a solid vector space, and let $a, b \in Y$ be two vectors with $a \prec b$. Then the set $(a, b)=\{x \in Y: a \prec x \prec b\}$ is called an open interval in $Y$.

It is easy to see that every open interval in $Y$ is an infinite set. Indeed, one can prove that $a+\lambda(b-a) \in(a, b)$ for all $\lambda \in \mathbb{R}$ with $0<\lambda<1$.

Theorem 6.1 Let $(Y, \preceq, \prec, \rightarrow)$ be a solid vector space. Then the collection $\mathcal{B}$ of all open intervals in $Y$ is a basis for a Hausdorff topology $\tau$ on $Y$.

Proof One has to prove that $\mathcal{B}$ satisfies the requirements for a basis. First, note that every vector $x$ of $Y$ lies in at least one element of $\mathcal{B}$. Indeed, $x \in(x-c, x+c)$ for each vector $c \succ 0$. Second, note that the intersection of any two open intervals contains another open interval, or is empty. Suppose $\left(a_{1}, b_{1}\right)$ and $\left(a_{2}, b_{2}\right)$ are two elements of $\mathcal{B}$ and a vector $x$ lies in their intersection. Then $b_{i}-x \succ 0$ and $x-a_{i} \succ 0$ for $i=1,2$. It follows from (S12) that there exists a vector $c \succ 0$ such that $c \prec b_{i}-x$ and $c \prec x-a_{i}$ for $i=1,2$. Hence, $a_{i} \prec x-c$ and $x+c \prec b_{i}$ for $i=1,2$. This implies that

$$
(x-c, x+c) \subset\left(a_{1}, b_{1}\right) \cap\left(a_{2}, b_{2}\right) .
$$

It remains to show that the topology $\tau$ is Hausdorff. We shall prove that for all $x, y \in X$ with $x \neq y$ there exists $c \succ 0$ such that the intersection of the intervals $(x-c, x+c)$ and $(y-c, y+c)$ is empty. Assume the contrary. Then there exists $x, y \in X$ with $x \neq y$ such that for every $c \succ 0$ the intersection of $(x-c, x+c)$ and $(y-c, y+c)$ is nonempty. Now let $c \succ 0$ 
be fixed. Hence, there is a vector $z \in Y$ satisfying $x-c \prec z \prec x+c$ and $y-c \prec z \prec y+c$. Therefore,

$$
-c \prec x-z \prec c \quad \text { and } \quad-c \prec z-y \prec c \text {. }
$$

Using (S10), we get

$$
-2 c \prec x-y \prec 2 c \text {. }
$$

Applying these inequalities to $\frac{1}{2} c$, we conclude that $x-y \prec c$ and $y-x \prec c$ for each $c \succ 0$. Now it follows from (S11) that $x \preceq y$ and $y \preceq x$, which is a contradiction since $x \neq y$.

Thanks to Theorem 6.1, we can give the following definition.

Definition 6.2 Let $(Y, \preceq, \prec, \rightarrow)$ be a solid vector space. The topology $\tau$ on $Y$ with basis formed by open intervals in $Y$ is called the order topology on $Y$.

Remark 6.1 Let $(Y, \preceq, \prec, \rightarrow)$ be a solid vector space. It follows from the proof of Theorem 6.1 that the collection

$$
\mathcal{B}^{\prime}=\{(x-c, x+c): x, c \in Y, c \succ 0\}
$$

is also a basis for the order topology $\tau$ on $Y$.

Theorem 6.2 Let $(Y, \preceq, \prec, \rightarrow)$ be a solid vector space and let $\tau$ be the order topology on $Y$. Then:

(i) For a sequence $\left(x_{n}\right)$ in $Y, x_{n} \stackrel{\tau}{\rightarrow} x$ if and only iffor every $c \succ 0$ there exists $N \in \mathbb{N}$ such that $x-c \prec x_{n} \prec x-c$ for all $n>N$.

(ii) For a sequence $\left(x_{n}\right)$ in $Y, x_{n} \rightarrow x$ implies $x_{n} \stackrel{\tau}{\rightarrow} x$.

Proof The first claim follows from Remark 6.1. Let $x_{n} \rightarrow x$ and $(a, b)$ be a neighborhood of $x$. From $a \prec x \prec b$ and (S5), we conclude that $x_{n} \in(a, b)$ for all but finitely many $n$. Hence, $x_{n} \stackrel{\tau}{\rightarrow} x$ which proves the second claim.

At the end of the next section, we shall prove that the converse of the statement (ii) of Theorem 6.2 holds true if and only if $Y$ is normal.

Theorem 6.3 If $(Y, \preceq, \prec, \rightarrow)$ is a solid vector space, then the convergence on $Y$ has the following properties.

(C6) Each convergent sequence in $Y$ has a unique limit.

(C7) Each convergent sequence in $Y$ is bounded.

Proof (C6) Let $\left(x_{n}\right)$ be a convergent sequence in $Y$. Assume that there are $x, y \in Y$ such that $x_{n} \rightarrow x$ and $x_{n} \rightarrow y$. It follows from Theorem 6.2 that $x_{n} \stackrel{\tau}{\rightarrow} x$ and $x_{n} \stackrel{\tau}{\rightarrow} y$. According to Theorem 6.1 the topology $\tau$ is Hausdorff. Now by the uniqueness of the limit of a convergent sequence in the Hausdorff topological space $(Y, \tau)$, we conclude that $x=y$.

(C7) Let $\left(x_{n}\right)$ be a convergent sequence in $Y$ and $x_{n} \rightarrow x$. By Theorem 6.2, $x_{n} \stackrel{\tau}{\rightarrow} x$. Choose an open interval $(a, b)$ which contains $x$. Then there exists a natural number $N$ 
such that $x_{n} \in(a, b)$ for all $n \geq N$. According to (S13), the set $\left\{a, b, x_{1}, \ldots, x_{N}\right\}$ is bounded in $Y$ which proves that $\left(x_{n}\right)$ is bounded.

\section{Minkowski functional on solid vector spaces}

In this section, using the Minkowski functional, we prove that the order topology on every solid vector space is normable. Also, we show that every normal solid vector space $Y$ is normable in the sense that there exists a norm $\|\cdot\|$ on $Y$ such that $x_{n} \rightarrow x$ if and only if $x_{n} \stackrel{\|\cdot\|}{\rightarrow} x$. Finally, we give a criterion for a normal vector space and show that the convergence of a sequence in normal solid vector space has the properties of the convergence in $\mathbb{R}$. This last result shows that the sandwich theorem plays an important role in solid vector spaces.

Definition 7.1 Let $Y$ be a real vector space. A subset $A$ of $Y$ is called:

(a) absorbing, if for all $x \in Y$ there exists $\lambda>0$ such that $x \in \lambda A$;

(b) balanced, if $\lambda A \subset A$ for every $\lambda \in \mathbb{R}$ with $|\lambda| \leq 1$.

Definition 7.2 Let $Y$ be a real vector space and $A \subset Y$ an absorbing set. Then the functional $\|\cdot\|: Y \rightarrow \mathbb{R}$ defined by

$$
\|x\|=\inf \{\lambda \geq 0: x \in \lambda A\}
$$

is called the Minkowski functional of $A$.

It is well known (see, e.g., Rudin [59, Theorem 1.35]) that the Minkowski functional of every absorbing, convex and balanced subset $A$ of a vector space $Y$ is a seminorm on $Y$.

Lemma 7.1 Let $(Y, \preceq, \rightarrow)$ be an ordered vector space with convergence. Suppose a subset $A$ of $Y$ is an absorbing, convex, balanced and bounded. Then the Minkowski functional $\|\cdot\|: Y \rightarrow \mathbb{R}$ of $A$ is a norm on $Y$. Moreover, if $A$ is closed, then

$$
\|x\|=\min \{\lambda \geq 0: x \in \lambda A\} .
$$

Proof Let $x \in Y$ be fixed and let $B_{x}=\{\lambda \geq 0: x \in \lambda A\}$. Since $A$ is absorbing, $B_{x}$ is nonempty. Since $A$ is balanced, $\alpha \in B_{x}$ and $\alpha<\beta$ imply $\beta \in B_{x}$. Let inf $B_{x}=\lambda$. By the definition of $\inf B_{x}$, for every $n \in \mathbb{N}$ there exists $\alpha \in B$ such that $\alpha<\lambda+\frac{1}{n}$. Hence, $\lambda+\frac{1}{n} \in B_{x}$ which means that $x \in\left(\lambda+\frac{1}{n}\right) A$.

Now we are ready to prove the $\|\cdot\|$ is indeed a norm on $Y$. Since the Minkowski functional of $A$ is seminorm, we have only to prove that $\|x\|=0$ implies $x=0$. Let $x$ be a vector in $Y$ such that $\|x\|=0$. In the case $\lambda=0$ the inclusion $x \in\left(\lambda+\frac{1}{n}\right) A$ reduces to $x \in \frac{1}{n} A$. Since $A$ is bounded, there is an interval $[a, b]$ containing $A$. Hence, $\frac{1}{n} a \preceq x \preceq \frac{1}{n} b$ for all $n \in \mathbb{N}$. According to (C3), $\frac{1}{n} a \rightarrow 0$ and $\frac{1}{n} b \rightarrow 0$. Hence, applying (V3) we conclude that $0 \preceq x \preceq 0$ which means that $x=0$.

It remains to prove (7.2) provided that $A$ is closed. We have to prove that $\lambda=\inf B_{x}$ belongs to $B_{x}$. If $\lambda=0$, then $x=0$ which implies that $\lambda \in B_{x}$. Now let $\lambda \neq 0$. The inclusion $x \in\left(\lambda+\frac{1}{n}\right) A$ implies that the sequence $\left(x_{n}\right)$ defined by $x_{n}=\left(\lambda+\frac{1}{n}\right)^{-1} x$ lies in $A$. According to (C3), $x_{n} \rightarrow \lambda^{-1} x$ which implies $x \lambda^{-1} \in A$ since $A$ is closed. Hence, $x \in \lambda A$ which proves that $\lambda \in B_{x}$. 
Definition 7.3 Let $(Y, \preceq, \rightarrow)$ be an ordered vector space with convergence, and let $a, b \in Y$ be two vectors with $a \preceq b$. The set $[a, b]=\{x \in Y: a \preceq x \preceq b\}$ is called a closed interval in $Y$.

Obviously, every closed interval $[-b, b]$ in an ordered vector space with convergence $Y$ is a convex, balanced, closed and bounded set. It follows from (S14) that $[-b, b]$ is also an absorbing set provided that $Y$ is a solid vector space and $b \succ 0$.

Definition 7.4 Let $(Y, \preceq, \rightarrow)$ be an ordered vector space with convergence. A norm $\|\cdot\|$ on $Y$ is called:

(a) monotone if $\|x\| \leq\|y\|$ whenever $0 \preceq x \preceq y$;

(b) semimonotone if there exists a constant $K>0$ such that $\|x\| \leq K\|y\|$ whenever $0 \preceq x \preceq y$.

Lemma 7.2 Let $(Y, \preceq, \prec, \rightarrow)$ be a solid vector space, and let $\|\cdot\|: Y \rightarrow \mathbb{R}$ be the Minkowski functional of $[-b, b]$ for some vector $b$ in $Y$ with $b \succ 0$. Then:

(i) $\|\cdot\|$ is a monotone norm on $Y$ which can be defined by

$$
\|x\|=\min \{\lambda \geq 0:-\lambda b \preceq x \preceq \lambda b\} .
$$

(ii) For $x \in Y$ and $\varepsilon>0$,

$$
\|x\|<\varepsilon \quad \text { if and only if }-\varepsilon b \prec x \prec \varepsilon b .
$$

Proof (i) The claim with the exception of the monotonicity of the norm follows from Lemma 7.1. Let $x$ and $y$ be two vectors in $Y$ such that $0 \preceq x \preceq y$. From (7.3), we get $y \preceq\|y\| b$. Hence, $-\|y\| b \preceq x \preceq\|y\| b$. Again from (7.3), we obtain $\|x\| \preceq\|y\|$. Hence, $\|\cdot\|$ is a monotone norm.

(ii) Let $\|x\|<\varepsilon$. From (7.3), we have $-\|x\| b \preceq x \preceq\|x\| b$, which implies

$$
-\varepsilon b \prec x \prec \varepsilon b .
$$

Conversely, let $-\varepsilon b \prec x \prec \varepsilon b$. Then $\varepsilon b-x \succ 0$ and $\varepsilon b+x \succ 0$. It follows from (S12) that there is $\lambda>0$ such that $\varepsilon b-x>\lambda b$ and $\varepsilon b+x>\lambda b$. Consequently,

$$
-(\varepsilon-\lambda) b \prec x \prec(\varepsilon-\lambda) b .
$$

From this and (7.3), we conclude that $\|x\| \leq \varepsilon-\lambda<\varepsilon$.

The following theorem shows that the order topology on $Y$ is normable.

Theorem 7.1 Let $(Y, \preceq, \prec, \rightarrow)$ be a solid vector space, and let $\|\cdot\|: Y \rightarrow \mathbb{R}$ be the Minkowski functional of $[-b, b]$ for some $b \in Y$ with $b \succ 0$. Then:

(i) The monotone norm $\|\cdot\|$ generates the order topology on $Y$.

(ii) For a sequence $\left(x_{n}\right)$ in $Y, x_{n} \rightarrow x$ implies $x_{n} \stackrel{\|\cdot\|}{\rightarrow} x$.

(iii) For a sequence $\left(x_{n}\right)$ in $Y, x_{n} \rightarrow x$ if and only if $x_{n} \stackrel{\|\cdot\|}{\rightarrow} x$, provided that the space $Y$ is normal. 
Proof (i) Denoting by $B(x, \varepsilon)$ an open ball in the normed space $(Y,\|\cdot\|)$, we shall prove that each $B(x, \varepsilon)$ contains some interval $(u, v)$ in $Y$ and vice versa. First, we shall prove the following identity:

$$
B(x, \varepsilon)=(x-\varepsilon b, x-\varepsilon b) \quad \text { for all } x \in Y \text { and } \varepsilon>0 .
$$

According to Lemma 7.2, for each $x, y \in Y$ and $\varepsilon>0$,

$$
\|x-y\|<\varepsilon \quad \text { if and only if } \quad x-\varepsilon b \prec y \prec x+\varepsilon b
$$

which proves (7.5). Note that identity (7.5) means that every open ball in the normed space $(Y,\|\cdot\|)$ is an open interval in $Y$. Now let $(u, v)$ be an arbitrary open interval in $Y$ and let $x \in(u, v)$. Choose an interval of the type $(x-c, x+c)$, which is a subset of $(u, v)$, where $c \in Y$ with $c \succ 0$. Then choosing $\varepsilon>0$ such that $\varepsilon b \prec c$, we conclude by (7.5) that $B(x, \varepsilon) \subset(u, v)$.

(ii) follows from (i) and Theorem 6.2.

(iii) Suppose that $Y$ is a normal vector space. According to (ii), we have to prove that $x_{n} \stackrel{\|\cdot\|}{\rightarrow} x$ implies $x_{n} \rightarrow x$. To prove this it sufficient to show that $\left\|x_{n}\right\| \rightarrow 0$ implies $x_{n} \rightarrow 0$. Let $\left(x_{n}\right)$ be a sequence in $Y$ such that $\left\|x_{n}\right\| \rightarrow 0$. By Lemma 7.2, we have

$$
-\left\|x_{n}\right\| b \preceq x_{n} \preceq\left\|x_{n}\right\| b \text { for all } n \text {. }
$$

Now applying the sandwich theorem, we conclude that $x_{n} \rightarrow 0$, which completes the proof.

The main part of Theorem 7.1 can be formulated in the following theorem.

Theorem 7.2 Let $(Y, \preceq, \prec, \rightarrow)$ be a solid vector space. Then there exists a monotone norm $\|\cdot\|$ on $Y$ such that the following statements hold true.

(i) The norm $\|\cdot\|$ generates the order topology on $Y$.

(ii) For a sequence $\left(x_{n}\right)$ in $Y, x_{n} \rightarrow x$ implies $x_{n} \stackrel{\|\cdot\|}{\rightarrow} x$.

(iii) If $Y$ is a normal vector space, then for a sequence $\left(x_{n}\right)$ in $Y, x_{n} \rightarrow x$ if and only if $x_{n} \stackrel{\|\cdot\|}{\rightarrow} x$.

In the next theorem, we shall give a criterion for a normal vector space. In particular, this theorem shows that every normal solid vector space $Y$ is normable in the sense that there exists a norm $\|\cdot\|$ on $Y$ such that $x_{n} \rightarrow x$ if and only if $x_{n} \stackrel{\|\cdot\|}{\rightarrow} x$. Analogous result for normability of normal topological vector space was proved by Vandergraft [60].

Theorem 7.3 Let $(Y, \preceq, \prec, \rightarrow)$ be a solid vector space. Then the following statements are equivalent:

(i) $Y$ is a normal vector space.

(ii) The convergence in $Y$ is generated by a monotone norm on $Y$.

(iii) The convergence in $Y$ is generated by the order topology on $Y$.

Proof (i) $\rightarrow$ (ii) Suppose $Y$ is a normal vector space. Let $\|\cdot\|: Y \rightarrow \mathbb{R}$ be the Minkowski functional of $[-b, b]$ for some vector $b$ in $Y$ with $b \succ 0$. According to Lemma 7.2, the 
Minkowski functional of $[-b, b]$ is a monotone norm on $Y$. We shall prove that the convergence in $Y$ is generated by this norm. We have to prove that for a sequence $\left(x_{n}\right)$ in $Y$, $x_{n} \rightarrow x$ if and only if $x_{n} \stackrel{\|\cdot\|}{\rightarrow} x$. Without loss of generality, we may assume that $x=0$. Then we have to prove that $x_{n} \rightarrow 0$ if and only if $\left\|x_{n}\right\| \rightarrow 0$. Taking into account Theorem 7.1, we have only to prove that $\left\|x_{n}\right\| \rightarrow 0$ implies $x_{n} \rightarrow 0$. Let $\left\|x_{n}\right\| \rightarrow 0$. By Lemma 7.2, we get

$$
-\left\|x_{n}\right\| b \preceq x_{n} \preceq\left\|x_{n}\right\| b \text { for all } n \text {. }
$$

It follows from axiom (C3) that $\left\|x_{n}\right\| b \rightarrow 0$. Then by the sandwich theorem, we conclude that $x_{n} \rightarrow 0$.

(ii) $\rightarrow$ (iii) Suppose the convergence in $Y$ is generated by a monotone norm $\|\cdot\|$ on $Y$, i.e., for a sequence $\left(x_{n}\right)$ in $Y, x_{n} \rightarrow x$ if and only if $x_{n} \stackrel{\|\cdot\|}{\rightarrow} x$. We shall prove that the convergence in $Y$ is generated by the order topology $\tau$ on $Y$. According to Theorem 6.2, it is sufficient to prove that for a sequence $\left(x_{n}\right)$ in $Y, x_{n} \stackrel{\tau}{\rightarrow} x$ implies $x_{n} \rightarrow x$. Again without loss of generality, we may assume that $x=0$. Let $x_{n} \stackrel{\tau}{\rightarrow} 0$. Let $\varepsilon>0$ be fixed. It follows from Theorem 6.2 that for every vector $c \succ 0$,

$$
-c \prec x_{n} \prec c
$$

for all sufficiently large $n$. From (7.6), we obtain $0 \prec c-x_{n} \prec 2 c$. By monotonicity of the norm, we conclude that $\left\|c-x_{n}\right\| \leq 2\|c\|$, which implies that $\left\|x_{n}\right\| \leq 3\|c\|$. Now choosing a vector $c \succ 0$ such that $\|c\|<\varepsilon / 3$, we obtain $\left\|x_{n}\right\|<\varepsilon$ for all sufficiently large $n$. Hence, $\left\|x_{n}\right\| \rightarrow 0$, which is equivalent to $x_{n} \rightarrow x$.

(iii) $\rightarrow$ (i) Suppose the convergence in $Y$ is generated by the order topology on $Y$. We shall prove that $Y$ is normal. Obviously, condition (4.3) in Definition 4.5 is equivalent to the following:

$$
0 \preceq x_{n} \preceq y_{n} \quad \text { for all } n \text { and } y_{n} \rightarrow 0 \text { imply } \quad x_{n} \rightarrow 0 .
$$

Let $\left(x_{n}\right)$ and $\left(y_{n}\right)$ be two sequences in $Y$ such that $0 \preceq x_{n} \preceq y_{n}$ for all $n$ and $y_{n} \rightarrow 0$. We have to prove that $x_{n} \rightarrow 0$. Let $c \succ 0$ be fixed. It follows from $y_{n} \rightarrow 0$ and (S5) that $y_{n} \prec c$ for all but finitely many $n$. From this and $0 \preceq x_{n} \preceq y_{n}$, we conclude that $-c \prec x_{n} \prec c$ for all sufficiently large $n$. Now it follows from Theorem 6.2 that $x_{n} \stackrel{\tau}{\rightarrow} x$ which is equivalent to $x_{n} \rightarrow 0$.

Note that Theorem 7.3 remains true if we replace in it 'monotone norm' by 'semimonotone norm'

The following theorem shows that the convergence in a normal solid vector space has the properties of the convergence in $\mathbb{R}$.

Theorem 7.4 If $(Y, \preceq, \prec, \rightarrow)$ is a normal solid vector space, then the convergence on $Y$ has the following additional properties:

(C8) Each subsequence of a convergent sequence converges to the same limit.

(C9) The convergence of a sequence and its limit do not depend on finitely many of its terms.

(C10) If $\lambda_{n} \rightarrow \lambda$ in $\mathbb{R}$ and $x_{n} \rightarrow x$, then $\lambda_{n} x_{n} \rightarrow \lambda x$. 
(C11) If $\lambda_{n} \rightarrow 0$ in $\mathbb{R}$ and $\left(x_{n}\right)$ is a bounded sequence in $Y$, then $\lambda_{n} x_{n} \rightarrow 0$.

(C12) If $\left(\lambda_{n}\right)$ is a bounded sequence in $\mathbb{R}$ and $x_{n} \rightarrow 0$, then $\lambda_{n} x_{n} \rightarrow 0$.

(C13) For each sequence $\left(x_{n}\right)$ in $Y, x_{n} \rightarrow x$ if and only if for every $c \succ 0$ there exists a natural number $N$ such that $x-c \prec x_{n} \prec x+c$ for all $n>N$.

Proof Let $\|\cdot\|$ be a norm on $Y$ that generates the convergence in $Y$. The existence of such norm follows from Theorem 7.3. The properties $\mathrm{C} 8)-(\mathrm{C} 10)$ are valid in any normed space. Property $(\mathrm{C} 13)$ follows from Theorems 6.2 and 7.3. The proofs of $(\mathrm{C} 11)$ and $(\mathrm{C} 12)$ are similar. We will prove only (C11). Since $\left(x_{n}\right)$ is bounded, there exist $a, b \in Y$ such that $a \preceq x_{n} \preceq b$ for all $n$. This implies

$$
\left|\lambda_{n}\right| a \preceq\left|\lambda_{n}\right| x_{n} \preceq\left|\lambda_{n}\right| b .
$$

By axiom (C3), we get $\left|\lambda_{n}\right| a \rightarrow 0$ and $\left|\lambda_{n}\right| b \rightarrow 0$. Applying the sandwich theorem to the inequalities (7.8), we conclude that $\left|\lambda_{n}\right| x_{n} \rightarrow 0$. Then by Theorem 7.3, we obtain $\left\|\left|\lambda_{n}\right| x_{n}\right\| \rightarrow 0$, that is, $\left\|\lambda_{n} x_{n}\right\| \rightarrow 0$. Again by Theorem 7.3, we conclude that $\lambda_{n} x_{n} \rightarrow 0$.

\section{Cone metric spaces and cone normed spaces}

In this section, we introduce the notions of cone metric spaces and cone normed spaces. Cone metric spaces were first introduced in 1934 by Kurepa [3]. Cone normed spaces were first introduced in 1936 by Kantorovich [18, 61]. For more on these abstract metric spaces, see the monograph of Collatz [4], the survey paper of Zabrejko [6] as well as the recent monograph of Rus, Petruşel and Petruşel [15] and references therein.

There are a lot of generalizations of the classical Banach fixed point principle in cone metric spaces (see Schröder [16, 17], Perov [62], Collatz [4, Chapter 2], Zabrejko [6], De Pascale, Marino and Pietramala [51], Rus, Petruşel and Şerban [7], Rus, Petruşel and Petruşel $[15$, Chapter 6] and references therein).

Definition 8.1 Let $X$ be a nonempty set, and let $(Y, \preceq, \rightarrow)$ be an ordered vector space with convergence. A vector-valued function $d: X \times X \rightarrow Y$ is said to be a cone metric on $Y$ if the following conditions hold:

(i) $d(x, y) \succeq 0$ for all $x, y \in X$ and $d(x, y)=0$ if and only if $x=y$;

(ii) $d(x, y)=d(x, y)$ for all $x, y \in X$;

(iii) $d(x, y) \preceq d(x, z)+d(z, y)$ for all $x, y, z \in X$.

The pair $(X, d)$ is called a cone metric space over $Y$. The elements of a cone metric space $X$ are called points.

Obviously, every metric space is a cone metric space over $\mathbb{R}$. In Section 9 we show that the theory of cone metric spaces over solid vector spaces is very close to the theory of the metric spaces.

Definition 8.2 Let $K$ be an arbitrary field. A map $|\cdot|: K \rightarrow \mathbb{R}$ is called a norm or absolute value on $K$ if it satisfies the following axioms:

(i) $|x| \geq 0$ for all $x \in X$ and $|x|=0$ if and only if $x=0$;

(ii) $|x y|=|x| \cdot|y|$ for all $x, y \in X$;

(iii) $|x+y| \leq|x|+|y|$ for all $x \in X$. 
An absolute value is called trivial if $|x|=1$ for $x \neq 0$. A field $(K,|\cdot|)$ equipped with a nontrivial absolute value is called a normed field or valued field.

Note that finite fields and their extensions only have the trivial absolute value. A normed field is always assumed to carry the topology induced by the metric

$$
\rho(x, y)=|x-y|
$$

with respect to which it is a topological field. An absolute value is also called a multiplicative valuation or a norm. For more on normed fields, see, e.g., Katok [63, Section 1.2] or Engler and Prestel [64, Section 1.1].

One of the most important class of cone metric spaces is the class of cone normed spaces.

Definition 8.3 Let $X$ be a vector space over a normed field $(\mathbb{K},|\cdot|)$, and let $(Y, \preceq, \rightarrow)$ be an ordered vector space with convergence. A map $\|\cdot\|: X \rightarrow Y$ is said to be a cone norm on $X$ if the following conditions hold:

(i) $\|x\| \succeq 0$ for all $x \in X$ and $\|x\|=0$ if and only if $x=0$;

(ii) $\|\lambda x\|=|\lambda|\|y\|$ for all $\lambda \in \mathbb{K}$ and $x \in X$;

(iii) $\|x+y\| \preceq\|x\|+\|y\|$ for all $x, y \in X$.

The pair $(X,\|\cdot\|)$ is said to be a cone normed space over $Y$.

It is easy to show that each cone normed space $(X,\|\cdot\|)$ over an ordered vector space with convergence $Y$ is a cone metric space over $Y$ with the cone metric defined by $d(x, y)=\|x-y\|$.

We end this section with the definitions of closed balls and bounded sets in cone metric spaces.

Definition 8.4 Suppose $(X, d)$ is a cone metric space over an ordered vector space with convergence $(Y, \preceq, \rightarrow)$. For a point $x_{0} \in X$ and a vector $r \in Y$ with $r \succeq 0$, the set

$$
\bar{U}\left(x_{0}, r\right)=\left\{x \in X: d\left(x, x_{0}\right) \preceq r\right\}
$$

is called a closed ball with center $x_{0}$ and radius $r$.

Definition 8.5 Let $X$ be a cone metric space.

(a) A set $A \subset X$ is called bounded if it is contained in some closed ball.

(b) A sequence $\left(x_{n}\right)$ in $X$ is called bounded if the set of its terms is bounded.

Let $(X, d)$ be a cone metric space over an ordered vector space with convergence $(Y, \preceq, \rightarrow)$. It is easy to show that a nonempty set $A \subset X$ is bounded if and only if there exists a vector $b \in Y$ such that $d(x, y) \preceq b$ for all $x, y \in A$.

Analogously, if $(X,\|\cdot\|)$ is a cone normed space over an ordered vector space with convergence $(Y, \preceq, \rightarrow)$, then a nonempty set $A \subset X$ is bounded if and only if there exists a vector $b \in Y$ such that $\|x\| \preceq b$ for all $x \in A$. 


\section{Cone metric spaces over solid vector spaces}

In this section, we shall study the cone metric spaces over solid vector spaces. The theory of such cone metric spaces is very close to the theory of the usual metric spaces. We show that every cone metric space over a solid vector space is a metrizable topological space. Every cone normed space over a solid vector space is normable.

\subsection{Topological structure of cone metric spaces}

Definition 9.1 Let $(X, d)$ be a cone metric space over a solid vector space $(Y, \preceq, \prec, \rightarrow)$. For a point $x_{0} \in X$ and a vector $r \in Y$ with $r \succ 0$, the set

$$
U\left(x_{0}, r\right)=\left\{x \in X: d\left(x, x_{0}\right) \prec r\right\}
$$

is called an open ball with center $x_{0}$ and radius $r$.

Theorem 9.1 Let $(X, d)$ be a cone metric space over a solid vector space $(Y, \preceq, \prec, \rightarrow)$. Then the collection

$$
\mathcal{B}=\{U(x, r): x \in X, r \in Y, r \succ 0\}
$$

of all open balls in $X$ is a basis for a topology $\tau_{d}$ on $X$.

Proof Suppose that $U\left(x_{1}, c_{1}\right)$ and $U\left(x_{2}, c_{2}\right)$ are two open balls in $X$ and take

$$
x \in U\left(x_{1}, c_{1}\right) \cap U\left(x_{2}, c_{2}\right) .
$$

Then $d\left(x, x_{i}\right) \prec c_{i}$ for $i=1$,2. From (S3), we get $c_{i}-d\left(x, x_{i}\right) \succ 0$ for $i=1$,2. It follows from (S12) that there exists a vector $c \in Y$ with $c \succ 0$ such that $c \prec c_{i}-d\left(x, x_{i}\right)$ for $i=1,2$. By (S3), we obtain $d\left(x, x_{i}\right) \prec c_{i}-c$ for $i=1,2$. Now using the triangle inequality and (S10), it easy to show that

$$
U(x, c) \subset U\left(x_{1}, c_{1}\right) \cap U\left(x_{2}, c_{2}\right) .
$$

Therefore, the collection $\mathcal{B}$ is a basis for a topology on $X$.

Thanks to Theorem 9.1 we can give the following definition.

Definition 9.2 Let $(X, d)$ be a cone metric space over a solid vector space $(Y, \preceq, \prec, \rightarrow)$. The topology $\tau_{d}$ on $X$ with basis formed by open balls in $X$ is called the cone metric topology on $X$.

We shall always assume that a cone metric space $(X, d)$ over a solid vector space $Y$ is endowed with the cone metric topology $\tau_{d}$. Hence, every cone metric space is a topological space.

Definition 9.3 Let $(X, d)$ be a cone metric space over a solid vector space $(Y, \preceq, \prec, \rightarrow)$. Then: 
(i) A sequence $\left(x_{n}\right)$ in $X$ is called Cauchy if for every $c \in Y$ with $c \succ 0$ there is $N \in \mathbb{N}$ such that $d\left(x_{n}, x_{m}\right) \prec c$ for all $n, m>N$.

(ii) A cone metric space $X$ is called complete if each Cauchy sequence in $X$ is convergent.

(iii) A complete cone normed space is called a cone Banach space.

In the following theorem, we show that each cone metric space $(X, d)$ over a solid vector space is metrizable. Besides, if $(X, d)$ is a complete cone metric space, then it is completely metrizable. Moreover, the cone metric is equivalent to a metric which preserve some inequalities.

Theorem 9.2 Let $(X, d)$ be a cone metric space over a solid vector space $(Y, \preceq, \prec, \rightarrow)$. Suppose $\|\cdot\|: Y \rightarrow \mathbb{R}$ is the Minkowski functional of $[-b, b]$ for some $b \in Y$ with $b \succ 0$. Define the metric $\rho$ on $X$ by $\rho(x, y)=\|d(x, y)\|$. Then:

(i) The topology of $(X, d)$ coincides with the topology of $(X, \rho)$.

(ii) $(X, d)$ is complete if and only if $(X, \rho)$ is complete.

(iii) For $x, x_{1}, \ldots, x_{n} \in X, y, y_{1}, \ldots, y_{n} \in X, a \in Y$ and $\lambda_{1}, \ldots, \lambda_{n} \in \mathbb{R}$,

$$
d(x, y) \preceq a+\sum_{i=1}^{n} \lambda_{i} d\left(x_{i}, y_{i}\right) \quad \text { implies } \quad \rho(x, y) \leq\|a\|+\sum_{i=1}^{n} \lambda_{i} \rho\left(x_{i}, y_{i}\right)
$$

Proof (i) It follows from Lemma 7.2(i) and Definition 8.1 that $\rho$ is a metric on $X$. Denoting by $B(x, \varepsilon)$ an open ball in the metric space $(X, \rho)$ and by $U(x, c)$ an open ball in the cone metric space $(X, d)$, we shall prove that each $B(x, \varepsilon)$ contains some $U(x, c)$ and vice versa. First, we shall show that

$$
B(x, \varepsilon)=U(x, \varepsilon b) \quad \text { for all } x \in X \text { and } \varepsilon>0 \text {. }
$$

According to Lemma 7.2(ii), for all $x, y \in X$ and $\varepsilon>0$,

$$
\|d(x, y)\|<\varepsilon \quad \text { if and only if } \quad d(x, y) \prec \varepsilon b \text {, }
$$

that is,

$$
\rho(x, y)<\varepsilon \quad \text { if and only if } \quad d(x, y) \prec \varepsilon b
$$

which proves (9.1). Note that identity (9.1) means that every open ball in the metric space $(X, \rho)$ is an open ball in the cone metric space $(X, d)$. Now let $U(x, c)$ be an arbitrary open ball in the cone metric space $(X, d)$. Choosing $\varepsilon>0$ such that $\varepsilon b \prec c$, we conclude by (9.1) that $B(x, \varepsilon) \subset U(x, c)$.

(ii) Let $\left(x_{n}\right)$ be a sequence in $X$. We have to prove that $\left(x_{n}\right)$ is $d$-Cauchy if and only if it is $\rho$-Cauchy. First note that (9.2) implies that for each $\varepsilon>0$ and all $m, n \in \mathbb{N}$,

$$
\rho\left(x_{n}, x_{m}\right)<\varepsilon \quad \text { if and only if } \quad d\left(x_{n}, x_{m}\right) \prec \varepsilon b \text {. }
$$

Let $\left(x_{n}\right)$ be $d$-Cauchy and $\varepsilon>0$ be fixed. Then there is an integer $N$ such that $d\left(x_{n}, x_{m}\right) \prec \varepsilon b$ for all $m, n>N$. Hence, $\rho\left(x_{n}, x_{m}\right)<\varepsilon$ for all $m, n>N$ which means that $\left(x_{n}\right)$ be $\rho$-Cauchy. 
Now, let $\left(x_{n}\right)$ be $\rho$-Cauchy and $c \succ 0$ be fixed. Choose $\varepsilon>0$ such that $\varepsilon b \prec c$. Then there is an integer $N$ such that $d\left(x_{n}, x_{m}\right)<\varepsilon$ for all $m, n>N$. Therefore, for these $n$ and $m$ we get $d\left(x_{n}, x_{m}\right) \prec \varepsilon b \prec c$, which means that $\left(x_{n}\right)$ is $d$-Cauchy.

(iii) follows from the monotony of the norm $\|\cdot\|$ and the definition of the metric $\rho$.

As we have seen the identity (9.1) plays an important role in the proof of Theorem 9.2. It is easy to see that this identity holds also for closed balls in the spaces $(X, \rho)$ and $(X, d)$. Namely, we have

$$
\bar{B}(x, \varepsilon)=\bar{U}(x, \varepsilon b) \quad \text { for all } x \in X \text { and } \varepsilon>0 .
$$

The main idea of Theorem 9.2 can be formulated in the following theorem.

Theorem 9.3 Let $(X, d)$ be a cone metric space over a solid vector space $(Y, \preceq, \prec, \rightarrow)$. Then there exists a metric $\rho$ on $X$ such that the following statements hold true.

(i) The topology of $(X, d)$ coincides with the topology of $(X, \rho)$.

(ii) $(X, d)$ is complete if and only if $(X, \rho)$ is complete.

(iii) For $x, x_{1}, \ldots, x_{n} \in X, y, y_{1}, \ldots, y_{n} \in X$ and $\lambda_{1}, \ldots, \lambda_{n} \in \mathbb{R}$,

$$
d(x, y) \preceq \sum_{i=1}^{n} \lambda_{i} d\left(x_{i}, y_{i}\right) \quad \text { implies } \quad \rho(x, y) \leq \sum_{i=1}^{n} \lambda_{i} \rho\left(x_{i}, y_{i}\right) .
$$

Metrizable topological spaces inherit all topological properties from metric spaces. In particular, it follows from Theorem 9.3 that every cone metric space over a solid vector space is a Hausdorff paracompact space and first-countable. Since every first countable space is sequential, we immediately get that every cone metric space is a sequential space. Hence, as a consequence of Theorem 9.3 we get the following corollary.

Corollary 9.1 Let $(X, d)$ be a cone metric space over a solid vector space $Y$. Then the following statements hold true:

(i) A subset of $X$ is open if and only if it is sequentially open.

(ii) A subset of $X$ is closed if and only if it is sequentially closed.

(iii) A function $f: D \subset X \rightarrow X$ is continuous if and only if it is sequentially continuous.

Lemma 9.1 Let $(X, d)$ be a cone metric space over a solid vector space $Y$. Then every closed ball $\bar{U}(a, r)$ in $X$ is a closed set.

Proof According to Corollary 9.1 we have to prove that $\bar{U}(a, r)$ is a sequentially closed set. Let $\left(x_{n}\right)$ be a convergent sequence in $\bar{U}(a, r)$ and let $x \in Y$ be its limit. Let $c \in Y$ with $c>0$ be fixed. Since $x_{n} \rightarrow x$, then there exists $n \in \mathbb{N}$ such that $d\left(x_{n}, x\right) \prec c$. Using the triangle inequality, we get $d(x, a) \preceq d\left(x_{n}, a\right)+d\left(x_{n}, x\right) \prec r+c$. Hence, $d(x, a)-r \prec c$ for all $c \in Y$ with $c \succ 0$. Then by (S11) we conclude that $d(x, a)-r \preceq 0$ which implies $x \in \bar{U}(a, r)$. Therefore, $\bar{U}(a, r)$ is a closed set in $X$.

Remark 9.1 Theorem 9.3 plays an important role in the theory of cone metric spaces over a solid vector space. In particular, using this theorem one can prove that some fixed point theorems in cone metric spaces are equivalent to their versions in usual metric spaces. For 
example, the short version of the Banach contraction principle in complete cone metric spaces (see Theorem 11.2 below) follows directly from its short version in metric spaces. $\mathrm{Du}$ [34] was the first who showed that there are equivalence between some metric and cone metric results. He obtained his results using the so-called nonlinear scalarization function. One year later, Kadelburg, Radenović and Rakočević [37] showed that the same results can be obtained using Minkowski functional in topological vector spaces.

Remark 9.2 Theorem 9.2 generalizes and extends some recent results of Du [34, Theorems 2.1 and 2.2], Kadelburg, Radenović and Rakočević [37, Theorems 3.1 and 3.2], Çakalli, Sönmez and Genç [40, Theorem 2.3], Simić [39, Theorem 2.2], Abdeljawad and Rezapour [43, Theorem 16] Arandelović and Kečkić [38, Lemma 2]. All of these authors have studied cone metric spaces over a solid Hausdorff topological vector space. Note that the identity (9.1) was proved by Çakalli, Sönmez and Genç [40, Theorem 2.2] provided that $Y$ is a Hausdorff topological vector space.

Theorem 9.2 generalizes and extends also some recent results of Amini-Harandi and Fakhar [23, Lemma 2.1], Turkoglu and Abuloha [26], Khani and Pourmahdian [29, Theorem 3.4], Sönmez [24, Theorem 1], Asadi, Vaezpour and Soleimani [30, Theorem 2.1], Feng and Mao [65, Theorem 2.2]. These authors have studied cone metric spaces over a solid Banach space.

Note that Asadi, Rhoades and Soleimani [41] proved that the metrics of Feng and Mao [65] and $\mathrm{Du}[34]$ are equivalent.

Finally, let us note a work of Khamsi [27] in which he introduced a metric-type structure in cone metric spaces over a normal Banach space.

Definition 9.4 Let $(X,\|\cdot\|)$ be a cone normed space over a solid vector space $Y$. The cone metric topology $\tau_{d}$ on $X$ induced by the metric $d(x, y)=\|x-y\|$ is called the cone topology on $X$.

In the following theorem, we show that each cone normed space $(X,\|\cdot\|)$ over a solid vector space is normable. Moreover, if $(X,\|\cdot\|)$ is a cone Banach space, then it is completely normable.

Theorem 9.4 Suppose $X$ is a vector space over a normed field $(\mathbb{K},|\cdot|)$. Let $(X,\|\cdot\|)$ be a cone normed space over a solid vector space $(Y, \preceq, \prec, \rightarrow)$. Let $\mu: Y \rightarrow \mathbb{R}$ be the Minkowski functional of $[-b, b]$ for some $b \in Y$ with $b \succ 0$. Define the norm $\|\cdot\| \mid \|$ by $\|x\|=\mu(\|\cdot\|)$. Then:

(i) The topology of $(X,\|\cdot\|)$ coincides with topology of $(X,\|\cdot\|)$.

(ii) $(X,\|\cdot\|)$ is a cone Banach space if and only if $(X,\|\cdot\| \|)$ is a Banach space.

(iii) For $x, x_{1}, \ldots, x_{n} \in X, a \in Y$ and $\lambda_{1}, \ldots, \lambda_{n} \in \mathbb{R}$,

$$
\|x\| \preceq a+\sum_{i=1}^{n} \lambda_{i}\left\|x_{i}\right\| \quad \text { implies } \quad\|x\| \leq \mu(a)+\sum_{i=1}^{n} \lambda_{i}\left\|x_{i}\right\| .
$$

Proof The topology of $(X,\|\cdot\|)$ is induced by the cone metric $d(x, y)=\|x-y\|$ and the topology of $(X,\|\cdot \mid\|)$ is induced by the metric $\rho(x, y)=\|x-y\|$. It is easy to see that $\rho=\mu \circ d$. Now the conclusions of the theorem follow from Theorem 9.2. 
Remark 9.3 Theorem 9.4(i) was recently proved by Çakalli, Sönmez and Genç [40, Theorem 2.4] provided that $\mathbb{K}=\mathbb{R}$ and $Y$ is a Hausdorff topological vector space.

The following corollary is an immediate consequence of Theorem 9.4(i).

Corollary 9.2 Every cone normed space $(X,\|\cdot\|)$ over a solid vector space $Y$ is a topological vector space.

\subsection{Convergence in cone metric spaces}

Let $(X, d)$ be a cone metric space over a solid vector space $(Y, \preceq, \prec, \rightarrow)$. Let $\left(x_{n}\right)$ be a sequence in $X$ and $x$ a point in $X$. We denote the convergence of $\left(x_{n}\right)$ to $x$ with respect to the cone metric topology, by $x_{n} \stackrel{d}{\rightarrow} x$ or simply by $x_{n} \rightarrow x$. Obviously, $x_{n} \stackrel{d}{\rightarrow} x$ if and only if for every vector $c \in Y$ with $c \succ 0, d\left(x_{n}, x\right) \prec c$ for all but finitely many $n$. This definition for the convergence in cone metric spaces over a solid Banach space can be found in the works of Chung [11,12] published in the period from 1981 to 1982. The definition of complete cone metric space (Definition 9.3) in the case when $Y$ is a solid Banach space also can be found in $[11,12]$.

Theorem 9.5 Let $(X, d)$ be a cone metric space over a solid vector space $Y$. Then the convergence in $X$ has the following properties:

(i) Any convergent sequence has a unique limit.

(ii) Any subsequence of a convergent sequence converges to the same limit.

(iii) Any convergent sequence is bounded.

(iv) The convergence and the limit of a sequence do not depend on finitely many of its terms.

Proof The properties (i), (ii) and (iv) are valid in any Hausdorff topological space. It remains to prove (iii). Let $\left(x_{n}\right)$ be a sequence in $X$ which converges to a point $x \in X$. Choose a vector $c_{1} \in Y$ with $c_{1} \succ 0$. Then there exists $N \in \mathbb{N}$ such that $d\left(x_{n}, x\right) \prec c_{1}$ for all $n \geq N$. By (S13), there is a vector $c_{2} \in Y$ such that $d\left(x_{n}, x\right) \prec c_{2}$ for all $n=1, \ldots, N$. Again by (S13), we get that there is a vector $c \in Y$ such that $c_{i} \prec c$ for $i=1,2$. Then by the transitivity of $\prec$, we conclude that $x_{n} \in U(x, c)$ for all $n \in \mathbb{N}$, which means that $\left(x_{n}\right)$ is bounded.

Applying Theorem 9.2, we shall prove a useful sufficient condition for convergence of a sequence in a cone metric space over a solid vector space.

Theorem 9.6 Let $(X, d)$ be a cone metric space over a solid vector space $(Y, \preceq, \prec, \rightarrow)$. Suppose $\left(x_{n}\right)$ is a sequence in $X$ satisfying

$$
d\left(x_{n}, x\right) \preceq b_{n}+\alpha d\left(y_{n}, y\right)+\beta d\left(z_{n}, z\right) \quad \text { for all } n,
$$

where $x$ is a point in $X,\left(b_{n}\right)$ is a sequence in $Y$ converging to $0,\left(y_{n}\right)$ is a sequence in $X$ converging to $y,\left(z_{n}\right)$ is a sequence in $X$ converging to $z$, $\alpha$ and $\beta$ are nonnegative numbers. Then the sequence $\left(x_{n}\right)$ converges to $x$. 
Proof Let $\|\cdot\|$ be the Minkowski functional of $[-b, b]$ for some $b \in Y$ with $b \succ 0$. Define the metric $\rho$ on $X$ as in Theorem 9.2. Then from (9.4), we get

$$
\rho\left(x_{n}, x\right) \preceq\left\|b_{n}\right\|+\alpha \rho\left(y_{n}, y\right)+\beta \rho\left(z_{n}, z\right) \text { for all } n \text {. }
$$

According to Theorem 7.1(ii), $b_{n} \rightarrow 0$ implies $\left\|b_{n}\right\| \rightarrow 0$. Hence, the right-hand side of (9.5) converges to 0 in $\mathbb{R}$. By the usual sandwich theorem, we conclude that $x_{n} \stackrel{\rho}{\rightarrow} x$ which is equivalent to $x_{n} \stackrel{d}{\rightarrow} x$.

Remark 9.4 A special case $(\alpha=\beta=0)$ of Theorem 9.6 was given without proof by Kadelburg, Radenović and Rakočević [42] in the case when $Y$ is a Banach space. This special case was proved by Şahin and Telsi [22, Lemma 3.3].

It is easy to see that if $\left(x_{n}\right)$ is a sequence in a cone metric space $(X, d)$ over a solid vector space $Y$, then

$$
d\left(x_{n}, x\right) \rightarrow 0 \text { implies } \quad x_{n} \stackrel{d}{\rightarrow} x
$$

but the converse is not true (see Example 9.2(ii) below). Note also that in general case the cone metric is not (sequentially) continuous function (see Example 9.2(iii) below), that is, from $x_{n} \rightarrow x$ and $y_{n} \rightarrow y$ it need not follow that $d\left(x_{n}, y_{n}\right) \rightarrow d(x, y)$.

In the following theorem, we shall prove that the converse of (9.6) holds provided that $Y$ is normal and solid.

Theorem 9.7 Suppose $(X, d)$ is a cone metric space over a normal solid vector space $(Y, \preceq$, $\prec, \rightarrow)$. Then

$$
x_{n} \stackrel{d}{\rightarrow} x \quad \text { if and only if } \quad d\left(x_{n}, x\right) \rightarrow 0
$$

Proof Let $\|\cdot\|$ be the Minkowski functional of $[-b, b]$ for some $b \in Y$ with $b \succ 0$. Define the metric $\rho$ on $X$ as in Theorem 9.2. By Theorem 9.2,

$$
x_{n} \stackrel{d}{\rightarrow} x \text { if and only if } \quad x_{n} \stackrel{\rho}{\rightarrow} x .
$$

By Theorems 7.1 and 7.3, for each sequence $\left(u_{n}\right)$ in $Y$

$$
u_{n} \rightarrow 0 \quad \text { if and only if } \quad\left\|u_{n}\right\| \rightarrow 0 \text {. }
$$

Applying this with $u_{n}=d\left(x_{n}, x\right)$, we get

$$
d\left(x_{n}, x\right) \rightarrow 0 \quad \text { if and only } \quad \rho\left(x_{n}, x\right) \rightarrow 0,
$$

that is,

$$
d\left(x_{n}, x\right) \rightarrow 0 \text { if and only } \quad x_{n} \stackrel{\rho}{\rightarrow} x .
$$

Now (9.7) follows from (9.8) and (9.9). 
The following theorem follows immediately from Corollary 9.2. It can also be proved by Theorem 9.6.

Theorem 9.8 Let $X$ be a vector space over a complete normed field $(\mathbb{K},|\cdot|)$, and let $(X,\|\cdot\|)$ be a cone normed space over a solid vector space $(Y, \preceq, \prec, \rightarrow)$. Then the convergence in $X$ satisfies the properties (i)-(iv) of Theorem 9.5 and it satisfies also the following properties:

(v) If $x_{n} \rightarrow x$ and $y_{n} \rightarrow y$, then $x_{n}+y_{n} \rightarrow x+y$.

(vi) If $\lambda_{n} \rightarrow \lambda$ in $\mathbb{K}$ and $x_{n} \rightarrow x$, then $\lambda_{n} x_{n} \rightarrow \lambda x$.

\subsection{Complete cone metric spaces}

Now we shall prove a useful sufficient condition for Cauchy sequence in cone metric spaces over a solid vector space. The second part of this result gives an error estimate for the limit of a convergent sequence in cone metric space. Also we shall prove a criterion for completeness of a cone metric space over a solid vector space.

Theorem 9.9 Let $(X, d)$ be a cone metric space over a solid vector space $(Y, \preceq, \prec, \rightarrow)$. Suppose $\left(x_{n}\right)$ is a sequence in $X$ satisfying

$$
d\left(x_{n}, x_{m}\right) \preceq b_{n} \text { for all } n, m \geq 0 \text { with } m \geq n,
$$

where $\left(b_{n}\right)$ is a sequence in $Y$ which converges to 0 . Then:

(i) The sequence $\left(x_{n}\right)$ is a Cauchy sequence in $X$.

(ii) If $\left(x_{n}\right)$ converges to a point $x \in X$, then

$$
d\left(x_{n}, x\right) \leq b_{n} \quad \text { for all } n \geq 0 .
$$

Proof (i) Let $c \in Y$ with $c \succ 0$ be fixed. According to (S5), $b_{n} \rightarrow 0$ implies that there exists $N \in \mathbb{N}$ such that $b_{n} \prec c$ for all $n>N$. It follows from (9.10) and (S2) that $d\left(x_{n}, x_{m}\right) \prec c$ for all $m, n>N$ with $m \geq n$. Therefore, $x_{n}$ is a Cauchy sequence in $X$.

(ii) Suppose $x_{n} \rightarrow x$. Let $n \geq 0$ be fixed. Choose an arbitrary $c \in Y$ with $c \succ 0$. Since $x_{n} \rightarrow x$, then there exists $m>n$ such that $d\left(x_{m}, x\right) \prec c$. By the triangle inequality, (9.10) and (S10), we get

$$
d\left(x_{n}, x\right) \preceq d\left(x_{n}, x_{m}\right)+d\left(x_{m}, x\right) \prec b_{n}+c .
$$

It follows from (S3) that $d\left(x_{n}, x\right)-b_{n} \prec c$ holds for each $c \succ 0$, which according to (S11) means that $d\left(x_{n}, x\right)-b_{n} \preceq 0$. Hence, $d\left(x_{n}, x\right) \preceq b_{n}$, which completes the proof.

Remark 9.5 The part (i) of Theorem 9.9 was proved by Azam, Beg and Arshad [36, Lemma 1.3] in the case when $Y$ is a topological vector space. Note also that whenever the cone metric space $(X, d)$ is complete, then the assumption of the second part of Theorem 9.9 is satisfied automatically.

A sequence of closed balls $\left(\bar{U}\left(x_{n}, r_{n}\right)\right)$ in a cone metric space $X$ is called a nested sequence if

$$
\bar{U}\left(x_{1}, r_{1}\right) \supset \bar{U}\left(x_{2}, r_{2}\right) \supset \cdots
$$


Now we shall prove a simple criterion for the completeness of a cone metric space over a solid vector space.

Theorem 9.10 (Nested ball theorem) A cone metric space $(X, d)$ over a solid vector space $(Y, \preceq, \prec, \rightarrow)$ is complete if and only if every nested sequence $\left(\bar{U}\left(x_{n}, r_{n}\right)\right)$ of closed balls in $X$ such that $r_{n} \rightarrow 0$ has a nonempty intersection.

Proof Let $\|\cdot\|$ be the Minkowski functional of $[-b, b]$ for some $b \in Y$ with $b \succ 0$. Define the metric $\rho$ on $X$ as in Theorem 9.2. By Theorem 9.2, $(X, d)$ is complete if and only if $(X, \rho)$ is complete.

Necessity. If $\left(\bar{U}\left(x_{n}, r_{n}\right)\right)$ is a nested sequence of closed balls in $(X, d)$ such that $r_{n} \rightarrow 0$, then according to Lemma 9.1 it is a nested sequence of closed sets in $(X, \rho)$ with the sequence of diameters $\left(\delta_{n}\right)$ converging to zero. Indeed, it easy to see that $\rho(x, y)=\|d(x, y)\| \preceq$ $2\left\|r_{n}\right\|$ for all $x, y \in \bar{U}\left(x_{n}, r_{n}\right)$. Hence, $\delta_{n} \leq 2\left\|r_{n}\right\|$, which yields $\delta_{n} \rightarrow 0$. Applying Cantor's intersection theorem to the metric space $(X, \rho)$, we conclude that the intersection of the sets $\bar{U}\left(x_{n}, r_{n}\right)$ is nonempty.

Sufficiently. Assume that every nested sequence of closed balls in $(X, d)$ with radii converging to zero has a nonempty intersection. We shall prove that each nested sequence $\left(\bar{B}\left(x_{n}, \varepsilon_{n}\right)\right)$ of closed balls in $(X, \rho)$ such that $\varepsilon_{n} \rightarrow 0$ has a nonempty intersection. By identity (9.3), we get

$$
\bar{B}\left(x_{n}, \varepsilon_{n}\right)=\bar{U}\left(x_{n}, r_{n}\right) \text { for all } n,
$$

where $r_{n}=\varepsilon_{n} b \rightarrow 0$. Hence, according to the assumptions the balls $\bar{B}\left(x_{n}, \varepsilon_{n}\right)$ have a nonempty intersection. Applying the nested ball theorem to the metric space $(X, \rho)$, we conclude that it is complete and so $(X, d)$ is also complete.

\subsection{Examples of complete cone metric spaces}

We end this section with three examples of complete cone metric spaces. Some other examples on cone metric spaces can be found in [6].

Example 9.1 Let $X$ be a nonempty set and let $(Y, \preceq, \prec, \rightarrow)$ be a solid vector space. Suppose $a$ is a vector in $Y$ such that $a \succeq 0$ and $a \neq 0$. Define the cone metric $d: X \times X \rightarrow Y$ by

$$
d(x, y)= \begin{cases}a & \text { if } x \neq y \\ 0 & \text { if } x=y\end{cases}
$$

Then $(X, d)$ is a complete cone metric space over $Y$. This space is called a discrete cone metric space.

Proof It is obvious that $(X, d)$ is a cone metric space (even if $Y$ is an arbitrary ordered vector space). We shall prove that every Cauchy sequence in $X$ is stationary. Assume the contrary and choose a sequence $\left(x_{n}\right)$ in $X$, which is Cauchy but not stationary. Then for every $c \in Y$ with $c \succ 0$ there exist $n, m \in \mathbb{N}$ such that $d\left(x_{n}, x_{m}\right) \prec c$ and $x_{n} \neq x_{m}$. Hence, $a \prec c$ for each $c \succ 0$. Then by (S11) we conclude that $a \preceq 0$ which together with $a \succeq 0$ leads to the contradiction $a=0$. Therefore, every Cauchy sequence in $X$ is stationary and so convergent in $X$. 
Example 9.2 Let $(Y, \preceq, \prec, \rightarrow)$ be a solid vector space, and let $X$ be its positive cone. Define the cone metric $d: X \times X \rightarrow Y$ as follows:

$$
d(x, y)= \begin{cases}x+y & \text { if } x \neq y \\ 0 & \text { if } x=y\end{cases}
$$

Then the following statements hold true:

(i) $(X, d)$ is a complete cone metric space over $Y$.

(ii) If $Y$ is not normal, then there are sequences $\left(x_{n}\right)$ in $X$ such that $x_{n} \rightarrow 0$ but $d\left(x_{n}, 0\right) \nrightarrow \rightarrow 0$.

(iii) If $Y$ is not normal, then the cone metric $d$ is not continuous.

Proof First we shall prove the following claim: A sequence $\left(x_{n}\right)$ in $X$ is Cauchy if and only if it satisfies one of the following two conditions:

(a) The sequence $\left(x_{n}\right)$ is stationary.

(b) For every $c \succ 0$ the inequality $x_{n} \prec c$ holds for all but finitely many $n$.

Necessity. Suppose $\left(x_{n}\right)$ is Cauchy but not stationary. Then for every $c \in Y$ with $c \succ 0$ there exists $N \in \mathbb{N}$ such that $d\left(x_{n}, x_{m}\right) \prec c$ for all $n, m>N$. Hence, for all $n, m>N$ we have $x_{n}+x_{m} \prec c$ whenever $x_{n} \neq x_{m}$. Let $n>N$ be fixed. Since $\left(x_{n}\right)$ is not stationary, there exists $m>N$ such that $x_{n} \neq x_{m}$. Hence, $x_{n}+x_{m} \prec c$. From this taking into account that $x_{m} \succeq 0$, we get $x_{n} \prec c$ and so $\left(x_{n}\right)$ satisfies (b).

Sufficiently. Suppose that $\left(x_{n}\right)$ satisfies (b). Then for every $c \succ 0$ there exists $N \in \mathbb{N}$ such that for all $n>N$ we have $x_{n} \prec \frac{1}{2} c$. Let $n, m>N$ be fixed. Then

$$
d\left(x_{n}, x_{m}\right) \preceq x_{n}+x_{m} \prec c
$$

which means that $\left(x_{n}\right)$ is Cauchy.

Now we shall prove the statements of the example.

(i) Let $\left(x_{n}\right)$ be a Cauchy sequence in $X$. If $\left(x_{n}\right)$ satisfies (a), then it is convergent. Now suppose that $\left(x_{n}\right)$ satisfies (b). Let $c \succ 0$ be fixed. Then $d\left(x_{n}, 0\right) \preceq x_{n} \prec c$ for all but finitely many $n$. This proves that $x_{n} \rightarrow 0$. Therefore, in both cases $\left(x_{n}\right)$ is convergent.

(ii) Since $Y$ is not normal, then there exist two sequences $\left(x_{n}\right)$ and $\left(y_{n}\right)$ in $Y$ such that $0 \preceq x_{n} \preceq y_{n}$ for all $n, y_{n} \rightarrow 0$ and $x_{n} \nrightarrow 0$. Let us consider $\left(x_{n}\right)$ as a sequence in $X$. It follows from the definition of the cone metric $d$ that $d\left(x_{n}, 0\right)=x_{n}$ for all $n$. Hence, $d\left(x_{n}, 0\right) \preceq y_{n}$ for all $n$. Then by Theorem 9.6, we conclude that $x_{n} \rightarrow 0$. On the other hand, $d\left(x_{n}, 0\right)=x_{n} \nrightarrow \supset 0$.

(iii) Assume that the cone metric $d$ is a continuous. Let $\left(x_{n}\right)$ be any sequence in $X$ satisfying (ii). From $x_{n} \rightarrow 0$ and continuity of the cone metric $d$, we obtain $d\left(x_{n}, 0\right) \rightarrow d(0,0)$, i.e. $x_{n} \rightarrow 0$ in $Y$ which is a contradiction. Hence, the cone metric $d$ is not continuous.

Example 9.3 Let $X=\mathbb{K}^{n}$ be $n$-dimensional vector space over $\mathbb{K}$, where $(\mathbb{K},|\cdot|)$ is a complete normed field. Let $Y=\mathbb{R}^{n}$ be equipped with the coordinate-wise convergence and the coordinate-wise ordering (see Example 5.1). Define the cone norm $\|\cdot\|: X \rightarrow Y$ by

$$
\|x\|=\left(\alpha_{1}\left|x_{1}\right|, \ldots, \alpha_{n}\left|x_{n}\right|\right)
$$


where $x=\left(x_{1}, \ldots, x_{n}\right)$ and $\alpha_{1}, \ldots, \alpha_{n}$ are positive numbers. Then $(X,\|\cdot\|)$ is a cone Banach space over $Y$.

\section{Iterated contractions in cone metric spaces}

The iterated contraction principle in usual metric spaces was briefly mentioned in 1968 by Rheinboldt [66] as a special case of a more general theorem. Two years later, the iterated contraction principle (with a posteriori error estimates) has been published in the famous monograph of Ortega and Rheinboldt [44, Theorem 12.3.2]. Great contribution to the iterated contraction principle in metric spaces and its applications to the fixed point theory was also given by Gel'man [67], Rus [68, 69], Kasahara [70], Hicks and Rhoades [71], Park [72] and others (see also Proinov [73] and [74, Section 6]). The iterated contraction principle is also known under the name of graphic contraction principle (see Rus, Petruşel and Petrușel [15, Section 3.2]).

In this section, we shall establish a full statement of the iterated contraction principle in cone metric spaces over a solid vector space. We shall formulate the result for nonselfmappings since the case of self-mappings is a special case of this one.

The following definition is due to Rheinbold [66] (see also Ortega and Rheinboldt [44, Definition 12.3.1]) in metric space setting.

Definition 10.1 Let $(X, d)$ be a cone metric space over an ordered vector space with convergence $(Y, \preceq, \rightarrow)$. A mapping $T: D \subset X \rightarrow X$ is said to be an iterated contraction on $D$ if there exists $0 \leq \lambda<1$ such that

$$
d\left(T x, T^{2} x\right) \preceq \lambda d(x, T x) \quad \text { for all } x \in D \text { with } T x \in D .
$$

The real number $\lambda$ is called a contractive constant of $T$.

Let $(X, d)$ be a cone metric space over a solid vector space $(Y, \preceq, \prec, \rightarrow)$, and let $T: D \subset X \rightarrow X$ be an arbitrary mapping in $X$. Then starting from a point $x_{0} \in D$ we can build up the Picard iterative sequence

$$
x_{n+1}=T x_{n}, \quad n=0,1,2, \ldots
$$

associated to the mapping $T$. We say that the iteration (10.2) is well defined if $x_{n} \in D$ for all $n=0,1,2, \ldots$. The main problems which arise for the Picard iteration are the following:

(i) Convergence problem. To find initial conditions for $x_{0} \in D$ which guarantee that the Picard iteration (10.2) is well defined and converging to a point $\xi \in D$.

(ii) Existence Problem. To find conditions which guarantee that $\xi$ is a fixed points of $T$.

(iii) Uniqueness Problem. To find a subset of $D$ in which $\xi$ is a unique fixed point of $T$.

(iv) Error estimates problem. To find a priory and a posteriori estimates for the cone distance $d\left(x_{n}, \xi\right)$.

In our opinion, the solving of problem (i) for the convergence of the Picard iteration plays an important role for the solving of problem (ii) for existence of fixed points of $T$. It turns out that in many cases the convergence of the Picard iteration to a point $\xi \in D$ 
implies that $\xi$ is a fixed point of $T$. For example, such situation can be seen in the next proposition.

Proposition 10.1 Let $(X, d)$ be a cone metric space over a solid vector space $(Y, \preceq, \prec, \rightarrow)$ and $T: D \subset X \rightarrow X$. Suppose the Picard iteration (10.2) for some $x_{0} \in D$ is well defined and converging to a point $\xi \in D$. Then each of the following conditions implies that $\xi$ is a fixed point of $T$.

(F1) $T$ is continuous at $\xi$.

(F2) Thas a closed graph.

(F3) $G(x)=\|d(x, T x)\|$ is lower semicontinuous at $\xi$ for some semimonotone norm $\|\cdot\|$ on $Y$.

(F4) $d(\xi, T \xi) \preceq \alpha d(x, \xi)+\beta d(T x, \xi)$ for each $x \in D$, where $\alpha, \beta \geq 0$.

Proof If either (F1) or (F2) is satisfied, then the conclusion follows from Theorem 9.5 and definition (10.2) of the Picard iteration.

Let the condition (F3) be satisfied. Since the norm $\|\cdot\|$ is semimonotone, there exists a constant $K>0$ such that $\|x\| \leq K\|y\|$ whenever $0 \preceq x \preceq y$. First we shall prove that $x_{n} \rightarrow \xi$ implies $\left\|d\left(x_{n}, x_{n+1}\right)\right\| \rightarrow 0$. We claim that for every $\varepsilon>0$ there exists a vector $c \in Y$ such that $c \succ 0$ and $\|c\|<\varepsilon$. To prove this take a vector $b \in Y$ with $b \succ 0$. We have $\left\|\frac{1}{n} b\right\|=\frac{1}{n}\|b\| \rightarrow 0$. Hence, every vector $c=\frac{1}{n} b$ with sufficiently large $n$ satisfies $\|c\|<\varepsilon$. Now let $\varepsilon>0$ be fixed. Choose a vector $c \in Y$ such that $c \succ 0$ and $\|c\|<\varepsilon / K$. From the triangle inequality, we get $d\left(x_{n}, x_{n+1}\right) \preceq d\left(x_{n}, \xi\right)+d\left(x_{n+1}, \xi\right)$. Now it follows from $x_{n} \rightarrow \xi$ that $d\left(x_{n}, x_{n+1}\right) \prec c$ for all but finitely many $n$. Hence, $\left\|d\left(x_{n}, x_{n+1}\right)\right\| \preceq K\|c\|<\varepsilon$ for these $n$. Therefore, $\left\|d\left(x_{n}, x_{n+1}\right)\right\| \rightarrow 0$. Now taking into account that $G$ is lower semicontinuous at $\xi$, we conclude that

$$
0 \leq\|d(\xi, T \xi)\|=G(\xi) \leq \liminf _{n \rightarrow \infty} G\left(x_{n}\right)=\liminf _{n \rightarrow \infty} \| d\left(x_{n}, x_{n+1} \|=0\right.
$$

which implies that $\xi$ is a fixed point of $T$.

Now suppose that the condition (F4) is satisfied. By substituting $x=x_{n}$, we get

$$
d(\xi, T \xi) \preceq \alpha d\left(x_{n}, \xi\right)+\beta d\left(x_{n+1}, \xi\right) .
$$

From this, taking into account that $x_{n} \rightarrow \xi$, we conclude that $d(\xi, T \xi) \prec c$ for each $c \in Y$ with $c \succ 0$. According to (S11), this implies $d(\xi, T \xi) \preceq 0$. Therefore, $d(\xi, T \xi)=0$, which means that $\xi$ is a fixed point of $T$.

Remark 10.1 Obviously, if the space $(X, d)$ in Proposition 10.1 is a metric space, then the function $G$ in (F4) can be defined by $G(x)=d(x, T x)$. In a metric space setting, this is a classical result (see [71]). Let us note also that if the space $Y$ in Proposition 10.1 is a normal solid normed space with norm $\|\cdot\|$, then one can choose in (F4) just this norm (see [20]).

Throughout this and the next section for convenience, we assume in $\mathbb{R}$ that $0^{0}=1$ by definition.

Proposition 10.2 Let $(X, d)$ be a cone metric space over a solid vector space $(Y, \preceq, \prec, \rightarrow)$ and $\left(x_{n}\right)$ is a sequence in $X$ satisfying

$$
d\left(x_{n+1}, x_{n+2}\right) \preceq \lambda d\left(x_{n}, x_{n+1}\right) \quad \text { for every } n \geq 0,
$$


where $0 \leq \lambda<1$. Then $\left(x_{n}\right)$ is a Cauchy sequence in $X$ and lies in the closed ball $\bar{U}\left(x_{0}, r\right)$ with radius

$$
r=\frac{1}{1-\lambda} d\left(x_{0}, x_{1}\right)
$$

Moreover, if $\left(x_{n}\right)$ converges to a point $\xi$ in $X$, then the following estimates hold:

$$
\begin{array}{ll}
d\left(x_{n}, \xi\right) & \preceq \frac{\lambda^{n}}{1-\lambda} d\left(x_{0}, x_{1}\right) \quad \text { for all } n \geq 0 ; \\
d\left(x_{n}, \xi\right) & \preceq \frac{1}{1-\lambda} d\left(x_{n}, x_{n+1}\right) \quad \text { for all } n \geq 0 \\
d\left(x_{n}, \xi\right) & \preceq \frac{\lambda}{1-\lambda} d\left(x_{n}, x_{n-1}\right) \quad \text { for all } n \geq 1 .
\end{array}
$$

Proof From (10.3) by induction on $n \geq 0$, we get

$$
d\left(x_{n}, x_{n+1}\right) \preceq \lambda^{n} d\left(x_{0}, x_{1}\right) \quad \text { for every } n \geq 0 .
$$

Now we shall show that $\left(x_{n}\right)$ satisfies

$$
d\left(x_{n}, x_{m}\right) \preceq b_{n} \quad \text { for all } n, m \geq 0 \text { with } m \geq n,
$$

where $b_{n}=\frac{\lambda^{n}}{1-\lambda} d\left(x_{0}, x_{1}\right)$. Indeed, for all $n, m \geq 0$ with $m \geq n$, we have

$$
\begin{aligned}
d\left(x_{n}, x_{m}\right) & \preceq \sum_{j=n}^{m} d\left(x_{j}, x_{j+1}\right) \preceq \sum_{j=n}^{m} \lambda^{j} d\left(x_{0}, x_{1}\right)=\left(\sum_{j=n}^{m} \lambda^{j}\right) d\left(x_{0}, x_{1}\right) \\
& \preceq\left(\sum_{j=n}^{\infty} \lambda^{j}\right) d\left(x_{0}, x_{1}\right)=\frac{\lambda^{n}}{1-\lambda} d\left(x_{0}, x_{1}\right)=b_{n} .
\end{aligned}
$$

It follows from axiom (C3) that $b_{n} \rightarrow 0$ in $Y$. Then by Theorem 9.9(i), we conclude that $\left(x_{n}\right)$ is a Cauchy sequence in $X$. Putting $n=0$ in (10.7) we obtain that $d\left(x_{m}, x_{0}\right) \preceq b_{0}$ for every $m \geq 0$. Hence, the sequence $\left(x_{n}\right)$ lies in the ball $\bar{U}\left(x_{0}, r\right)$ since $r=b_{0}$. Now suppose that $\left(x_{n}\right)$ converges to a point $\xi \in X$. Then it follows from Theorem 9.9(ii) that $\left(x_{n}\right)$ satisfies the inequality $d\left(x_{n}, \xi\right) \preceq b_{n}$ (for every $n \geq 0$ ) which proves (10.4). Applying (10.4) with $n=0$, we conclude that the first two terms of the sequence $\left(x_{n}\right)$ satisfy the inequality

$$
d\left(x_{0}, \xi\right) \preceq \frac{1}{1-\lambda} d\left(x_{0}, x_{1}\right) .
$$

Note that for every $n \geq 0$ the sequence $\left(x_{n}, x_{n+1}, x_{n+2}, \ldots\right)$ also satisfies (10.3) and converges to $\xi$. Therefore, applying the last inequality to the first two terms of this sequence we get (10.5). The inequality (10.6) follows from (10.5) and (10.3).

Remark 10.2 Proposition 10.2 generalizes, improves and complements a recent result of Latif and Shaddad [25, Lemma 3.1]. They have proved that a sequence $\left(x_{n}\right)$ in a cone metric space $X$ satisfying (10.3) is Cauchy provided that $Y$ is a normal Banach space. 
Theorem 10.1 (Iterated contraction principle) Let $(X, d)$ be a complete cone metric space over a solid vector space $(Y, \preceq, \prec, \rightarrow)$. Let $T: D \subset X \rightarrow X$ be a mapping satisfying the following conditions:

(a) $T$ is an iterated contraction on $D$ with contractive constant $\lambda<1$.

(b) There is $x_{0} \in D$ such that $\bar{U}\left(x_{0}, r\right) \subset D$, where $r=\frac{1}{1-\lambda} d\left(x_{0}, T x_{0}\right)$.

Then the following hold true:

(i) Convergence of the iterative method. The Picard iteration (10.2) starting from $x_{0}$ is well defined, remains in the closed ball $\bar{U}\left(x_{0}, r\right)$ and converges to a point $\xi \in \bar{U}\left(x_{0}, r\right)$.

(ii) A PRIORI ERROR ESTIMATE. The following estimate holds:

$$
d\left(x_{n}, \xi\right) \preceq \frac{\lambda^{n}}{1-\lambda} d\left(x_{0}, T x_{0}\right) \quad \text { for all } n \geq 0
$$

(iii) A posteriori error estimates. The following estimates hold:

$$
\begin{aligned}
& d\left(x_{n}, \xi\right) \preceq \frac{1}{1-\lambda} d\left(x_{n}, x_{n+1}\right) \quad \text { for all } n \geq 0 \text {; } \\
& d\left(x_{n}, \xi\right) \preceq \frac{\lambda}{1-\lambda} d\left(x_{n}, x_{n-1}\right) \quad \text { for all } n \geq 1 \text {. }
\end{aligned}
$$

(iv) EXISTENCE OF FIXED POINTS. If at least one of the conditions (F1)-(F2) is satisfied, then $\xi$ is a fixed point of $T$.

Proof Define the function $\rho: D \rightarrow Y$ by $\rho(x)=\frac{1}{1-\lambda} d(x, T x)$. It follows from condition (a) that $\rho(T x) \preceq \lambda \rho(x)$ for each $x \in D$ with $T x \in D$. Now define the set $U$ as follows

$$
U=\{x \in D: \bar{U}(x, \rho(x)) \subset D\} .
$$

It follows from $\rho\left(x_{0}\right)=r$ and (b) that the set $U$ is not empty. We shall prove that $T(U) \subset U$. Let $x$ be a given point in $U$. It follows from the definition of $\rho$ that $d(x, T x) \preceq \rho(x)$ which means that $T x \in \bar{U}(x, \rho(x)) \subset D$. Therefore, $T x \in D$. Further, we shall show that

$$
\bar{U}(T x, \rho(T x)) \subset \bar{U}(x, \rho(x)) .
$$

Indeed, suppose that $y \in \bar{U}(T x, \rho(T x))$. Then

$$
d(y, x) \preceq d(y, T x)+d(x, T x) \preceq \rho(T x)+d(x, T x) \preceq \lambda \rho(x)+d(x, T x)=\rho(x)
$$

which means that $y \in \bar{U}(x, \rho(x))$. Hence, $\bar{U}(T x, \rho(T x)) \subset D$ and so $T x \in U$. This proves that $T(U) \subset U$ which means that Picard iteration $\left(x_{n}\right)$ is well defined. From (a), we deduce that it satisfies (10.3). Now conclusions (i)-(iii) follow from Proposition 10.2. Conclusion (iv) follows from Proposition 10.1.

Remark 10.3 Obviously, whenever $T$ is a self-mapping of $X$, condition (b) of Theorem 10.1 is satisfied automatically for every $x_{0} \in X$ and so it can be omitted. If $D$ is closed and $T(D) \subset D$, then condition (b) also can be dropped. 
Remark 10.4 Note that Theorem 10.1(i) generalizes and extends some results of Pathak and Shahzad [21, Theorem 3.7] and Wardowski [20, Theorem 3.3]. Their results have been proved for a selfmapping $T$ of $X$ in the case when $Y$ is a normal Banach space.

\section{Contraction mappings in cone metric spaces}

In 1922, the famous Polish mathematician, Stefan Banach [75], established his famous fixed point theorem nowadays known as the Banach fixed point theorem or the Banach contraction principle. The Banach contraction principle is one of the most useful theorem in the fixed point theory. It has a short version and full version. Its full version in a metric space setting can be seen, for example, in the monographs of Kirk [76], Zeidler [57, Section 1.6] and Berinde [77, Section 2.1].

The object of this section is to give a full statement of the Banach contraction principle for a nonself-mapping in a cone metric spaces over a solid vector space.

Definition 11.1 ([75]) Let $(X, d)$ be a cone metric space over an ordered vector space with convergence $(Y, \preceq, \rightarrow)$. A mapping $T: D \subset X \rightarrow X$ is said to be a contraction on $D$ if there exists $0 \leq \lambda<1$ such that

$$
d(T x, T y) \preceq \lambda d(x, T y) \quad \text { for all } x, y \in D .
$$

The real number $\lambda$ is called a contractive constant of $T$.

Theorem 11.1 (Banach contraction principle) Let $(X, d)$ be a complete cone metric space over a solid vector space $(Y, \preceq, \prec, \rightarrow)$. Let $T: D \subset X \rightarrow X$ be a mapping satisfying the following conditions:

(a) $T$ is a contraction on $D$ with contractive constant $\lambda<1$.

(b) There is $x_{0} \in D$ such that $\bar{U}\left(x_{0}, r\right) \subset D$, where $r=\frac{1}{1-\lambda} d\left(x_{0}, T x_{0}\right)$.

Then the following hold true:

(i) Existence And uniQueness. $T$ has a unique fixed point $\xi$ in $D$.

(ii) Convergence of the iterative method. The Picard iteration (10.2) starting from $x_{0}$ is well defined, remains in the closed ball $\bar{U}\left(x_{0}, r\right)$ and converges to $\xi$.

(iii) A PRIORI ERROR ESTIMATE. The following estimate holds:

$$
d\left(x_{n}, \xi\right) \preceq \frac{\lambda^{n}}{1-\lambda} d\left(x_{0}, T x_{0}\right) \quad \text { for all } n \geq 0 .
$$

(iv) A POSTERIORI ERror estimates. The following estimates hold:

$$
\begin{aligned}
& d\left(x_{n}, \xi\right) \preceq \frac{1}{1-\lambda} d\left(x_{n}, x_{n+1}\right) \quad \text { for all } n \geq 0 \text {; } \\
& d\left(x_{n}, \xi\right) \preceq \frac{\lambda}{1-\lambda} d\left(x_{n}, x_{n-1}\right) \quad \text { for all } n \geq 1 \text {. }
\end{aligned}
$$

(v) RATE OF CONVERGENCE. The rate of convergence of the Picard iteration is given by

$$
\begin{array}{ll}
d\left(x_{n+1}, \xi\right) \preceq \lambda d\left(x_{n}, \xi\right) & \text { for all } n \geq 1 \\
d\left(x_{n}, \xi\right) \preceq \lambda^{n} d\left(x_{0}, \xi\right) & \text { for all } n \geq 0 .
\end{array}
$$


Proof Using the triangle inequality and the contraction condition (a), one can see that condition (F4) holds with $\alpha=\lambda$ and $\beta=1$. Conclusions (i)-(iv) with the exception of the uniqueness of the fixed point follow immediately from Theorem 10.1 since every contraction mapping is an iterated contraction mapping. Suppose $T$ has two fixed points $x, y \in D$. Then it follows from (a) that $d(x, y) \preceq \lambda d(x, y)$, which leads to $(1-\lambda) d(x, y) \preceq 0$ and so $d(x, y) \preceq 0$. Hence, $d(x, y)=0$ which means that $x=y$. Therefore, $\xi$ is a unique fixed point of $T$ in $D$. Conclusion (v) follows from (a) and (i) by putting $x=x_{n}$ and $y=\xi$.

Kirk in his paper [76] wrote for the Banach contraction principle in usual metric spaces the following, 'The great significance of Banach's principle, and the reason it is one of the most frequently cited fixed point theorems in all of analysis, lies in the fact that (i)-(v) contain elements of fundamental importance to the theoretical and practical treatment of mathematical equations'. We would add that in general cone metrics give finer estimates than usual metrics.

Recall that a self-mapping $T$ of a cone metric space $(X, d)$ is called contraction on $X$ if there exists $0 \leq \lambda<1$ such that $d(T x, T y) \preceq \lambda d(x, y)$ for all $x, y \in X$. The following short version of the Banach contraction principle for self-mappings in cone metric spaces follows immediately from Theorem 11.1. Note that the short version of Banach's principle follows also from the short version of Banach's principle in metric spaces and Theorem 9.2.

Theorem 11.2 Each contraction $T$ on a cone metric space $(X, d)$ over a solid vector space $Y$ has a unique fixed point and for each $x_{0} \in X$ the Picard iteration (10.2) converges to the fixed point.

Remark 11.1 Theorem 11.2 was proved by Huang and Zhang [13, Theorem 1] in the case when $Y$ is a normal Banach space. One year later, Rezapour and Hamlbarani [19, Theorem 2.3] improved their result omitting the assumption of normality. Finally, Du [34, Theorem 2.3] proved this result assuming that $Y$ is a locally convex Hausdorff topological vector space.

Remark 11.2 Recently, Radenović and Kadelburg [28, Theorem 3.3] have established the a priory estimate (11.2) for a self-mappings $T$ of a cone metric space $X$ over a solid Banach space $Y$.

Remark 11.3 In Section 8, we have mentioned that there are various generalizations of the Banach contraction principle in a cone metric space $(X, d)$ over an ordered vector $\mathcal{L}$-space $(Y, \preceq, \rightarrow)$. Many authors have obtained fixed point theorems for selfmappings $T: X \rightarrow X$ of the type

$$
d(T x, T y) \preceq Q d(x, T y) \quad \text { for all } x, y \in X,
$$

where $Q: Y_{+} \rightarrow Y_{+}$is a linear nonnegative operator. Obviously, condition (11.7) has more general form than (11.1). Nevertheless, Theorem 11.2 is not a consequence of the results mentioned in Section 2 because they are obtained under different assumptions. In particular, the authors of these results assume, by definition, that a sequence $\left(x_{n}\right)$ in $X$ converges to $x \in X$ if and only if $d\left(x_{n}, x\right) \rightarrow 0$ in $Y$, which is not true in cone metric spaces over an arbitrary solid vector space (see Section 9.2). 


\section{Conclusion}

In the first part of this paper (Sections 2-7), we develop a unified theory for solid vector spaces. A real vector space $Y$ with convergence $(\rightarrow)$ is called a solid vector space if it is equipped with a vector ordering $(\preceq)$ and a strict vector ordering $(\prec)$. It turns out that every convergent sequence in a solid vector space has a unique limit. Every solid vector space $Y$ can be endowed with an order topology $\tau$ such that $x_{n} \rightarrow x$ implies $x_{n} \stackrel{\tau}{\rightarrow} x$. It turns out that the converse of this implication holds if and only if the space $Y$ is normal, i.e., the sandwich theorem holds in $Y$. Using the Minkowski functional, we show that the order topology on every solid vector space is normable with a monotone norm. Among the other results in this part of the paper, we show that an ordered vector space can be equipped with a strict vector ordering if and only if it has a solid positive cone. Moreover, if the positive cone of the vector ordering is solid, then there exists a unique strict vector ordering on this space.

In the second part of the paper (Sections 8-9), we develop a unified theory for cone metric spaces and cone normed spaces over a solid vector space. We show that every (complete) cone metric space $(X, d)$ over a solid vector space $Y$ is a (completely) metrizable topological space. Moreover, there exists an equivalent metric $\rho$ on $X$ that preserve some inequalities. In particular, an inequality of the type

$$
d(x, y) \preceq \sum_{i=1}^{n} \lambda_{i} d\left(x_{i}, y_{i}\right) \quad\left(x, y, x_{i}, y_{i} \in X, \lambda_{i} \in \mathbb{R}\right)
$$

implies the inequality

$$
\rho(x, y) \preceq \sum_{i=1}^{n} \lambda_{i} \rho\left(x_{i}, y_{i}\right)
$$

Using this result, one can prove that some fixed point theorems in cone metric spaces are equivalent to their versions in usual metric spaces. For example, the short version of the Banach contraction principle in a cone metric space is equivalent to its version in a metric space because the Banach contractive condition $d(T x, T y) \preceq \lambda d(x, y)$ is of the type (12.1). Let us note that the above mentioned result cannot be applied to many contractive conditions in a cone metric space. That is why we need further properties of cone metric spaces. Further, we give some useful properties of cone metric spaces, which allow us to prove convergence results for Picard iteration with a priori and a posteriori error estimates. Among the other results in this part of the paper, we prove that every cone normed space over a solid vector space is normable.

In the third part of the paper (Sections 10-11), applying the cone metric theory, we present full statements of the iterated contraction principle and the Banach contraction principle in cone metric spaces over a solid vector space.

Let us note that some of the results of the paper (Theorems 9.2, 9.3, 9.4, 9.6 and 9.9; Propositions 10.1 and 10.2) give a method for obtaining convergence theorems (with error estimates) for Picard iteration and fixed point theorems in a cone metric space over a solid vector space.

Finally, let us note that we have come to the idea of a general theory of cone metric spaces (over a solid vector spaces) dealing with convergence problems of some iterative methods 
for finding all zeros of a polynomial $f$ simultaneously (i.e., as a vector in $\mathbb{C}^{n}$, where $n$ is the degree of $f$ ). In our next papers, we will continue studying the cone metric space theory and its applications. For instance, we shall show that almost all results given in Proinov [74, 78] can be extended in cone metric spaces over a solid vector space. Also, we shall present new convergence theorems for some iterative methods for finding zeros of a polynomial simultaneously. These results generalize, improve and complement a lot of results given in the monographs of Sendov, Andreev, Kjurkchiev [79] and Petković [80]. In particular, it turns out that the cone norms in $\mathbb{C}^{n}$ give better a priori and a posteriori error estimates for iterative methods in $\mathbb{C}^{n}$ than usual norms.

\section{Competing interests}

The author declares that he has no competing interests.

Received: 7 August 2012 Accepted: 4 April 2013 Published: 18 April 2013

\section{References}

1. Fréchet, M: La notion d'écart et le calcul fonctionnel. C. R. Math. Acad. Sci. Paris 140, 772-774 (1905)

2. Fréchet, M: Sur quelques points du calcul fonctionnel. Rend. Circ. Mat. Palermo 22, 1-74 (1906)

3. Kurepa, ĐR: Tableaux ramifiés d'ensembles. Espaces pseudo-distanciés. C. R. Math. Acad. Sci. Paris 198, 1563-1565 (1934)

4. Collatz, L: Functional Analysis and Numerical Mathematics. Academic Press, New York (1966)

5. Eisenfeld, J, Lakshmikantham, V: Comparison principle and nonlinear contractions in abstract spaces. J. Math. Anal. Appl. 49, 504-511 (1975)

6. Zabrejko, PP: K-metric and K-normed linear spaces: survey. Collect. Math. 48, 825-859 (1997)

7. Rus, IA, Petruşel, A, Şerban, MA: Weakly Picard operators: equivalent definitions, applications and open problems. Fixed Point Theory 7, 3-22 (2006)

8. Rzepecki, B: On fixed point theorems of Maia type. Publ. Inst. Math. (Belgr.) 28, 179-186 (1980)

9. Filip, AD, Petruşel, A: Fixed point theorems on spaces endowed with vector-valued metrics. Fixed Point Theory Appl. 2010, Article ID 281381 (2010). doi:10.1155/2010/281381

10. Agarwal, RP, Khamsi, MA: Extension of Caristi's fixed point theorem to vector valued metric spaces. Nonlinear Anal. 74, 141-145 (2011). doi:10.1016/j.na.2010.08.025

11. Chung, KJ: Nonlinear contractions in abstract spaces. Kodai Math. J. 4, 288-292 (1981)

12. Chung, KJ: Remarks on nonlinear contractions. Pac. J. Math. 101, 41-48 (1982)

13. Huang, LG, Zhang, X: Cone metric spaces and fixed point theorems of contractive mappings. J. Math. Anal. Appl. 332, 1468-1476 (2007). doi:10.1016/j.jmaa.2005.03.087

14. Janković, S, Kadelburg, Z, Radenović, S: On cone metric spaces: a survey. Nonlinear Anal. 74, 2591-2601 (2011). doi:10.1016/j.na.2010.12.014

15. Rus, IA, Petruşel, A, Petruşel, G: Fixed Point Theory. Cluj University Press, Cluj-Napoca (2008)

16. Schröder, J: Das Iterationsverfahren bei allgemeinerem Abstandsbegriff. Math. Z. 66, 111-116 (1956)

17. Schröder, J: Nichtlineare Majoranten beim Verfahren der schrittweisen Näherung. Arch. Math. 7, 471-484 (1956)

18. Kantorovich, LV: The method of successive approximations for functional equations. Acta Math. 71, 63-97 (1939)

19. Rezapour, S, Hamlbarani, R: Some notes on the paper: 'Cone metric spaces and fixed point theorems of contractive mappings'. J. Math. Anal. Appl. 345, 719-724 (2008). doi:10.1016/j.jmaa.2008.04.049

20. Wardowski, D: Endpoints and fixed points of set-valued contractions in cone metric spaces. Nonlinear Anal. 71, 512-516 (2009). doi:10.1016/j.na.2008.10.089

21. Pathak, HK, Shahzad, N: Fixed point results for generalized quasicontraction mappings in abstract metric spaces. Nonlinear Anal. 71, 6068-6076 (2009) doi:10.1016/j.na.2009.05.052

22. Şahin, I, Telci, M: A theorem on common fixed points of expansion type mappings in cone metric spaces. An. Univ. "Ovidius" Constanţa, Ser. Mat., 18, 329-336 (2010)

23. Amini-Harandi, A, Fakhar, M: Fixed point theory in cone metric spaces obtained via the scalarization method. Comput. Math. Appl. 59, 3529-3534 (2010). doi:10.1016/j.camwa.2010.03.046

24. Sönmez, A: On paracompactness in cone metric spaces. Appl. Math. Lett. 23, 494-497 (2010). doi:10.1016/j.aml.2009.12.011

25. Latif, A, Shaddad, FY: Fixed point results for multivalued maps in cone metric spaces. Fixed Point Theory Appl. 2010, Article ID 941371 (2010). doi:10.1155/2010/941371

26. Turkoglu, D, Abuloha, M: Cone metric spaces and fixed point theorems in diametrically contractive mappings. Acta Math. Sin. Engl. Ser. 26, 489-496 (2010). doi:10.1007/s10114-010-8019-5

27. Khamsi, MA: Remarks on cone metric spaces and fixed point theorems of contractive mappings. Fixed Point Theory Appl. 2010, Article ID 315398 (2010). doi:10.1155/2010/315398

28. Radenović, S, Kadelburg, Z: Quasi-contractions on symmetric and cone symmetric spaces. Banach J. Math. Anal. 5 , 38-50 (2011)

29. Khani, M, Pourmahdian, M: On the metrizability of cone metric spaces. Topol. Appl. 158, 190-193 (2011). doi:10.1016/j.topol.2010.10.016

30. Asadi, M, Vaezpour, SM, Soleimani, H: Metrizability of cone metric spaces (2011). arXiv:1 102.2353

31. Ding, H, Kadelburg, Z, Karapinar, E, Radenović, S: Common fixed points of weak contractions in cone metric spaces. Abstr. Appl. Anal. 2012, Article ID 793862 (2012). doi:10.1155/2012/793862 
32. Lin, IJ, Chen, CM, Jovanović, M, Wu, TH: Fixed points and endpoints of set-valued contractions in cone metric spaces. Abstr. Appl. Anal. 2012, Article ID 632628 (2012). doi:10.1155/2012/632628

33. Beg, I, Azam, A, Arshad, M: Common fixed points for maps on topological vector space valued cone metric spaces. Int. J. Math. Math. Sci. 2009, Article ID 560264 (2009). doi:10.1155/2009/560264

34. Du, WS: A note on cone metric fixed point theory and its equivalence. Nonlinear Anal. 72, 2259-2261 (2010). doi:10.1016/..na.2009.10.026

35. Du, WS: New cone fixed point theorems for nonlinear multivalued maps with their applications. Appl. Math. Lett. 24, 172-178 (2011). doi:10.1016/j.aml.2010.08.040

36. Azam, A, Beg, I, Arshad, M: Fixed point in topological vector space-valued cone metric spaces. Fixed Point Theory Appl. 2010, Article ID 604084 (2010). doi:10.1155/2010/604084

37. Kadelburg, Z, Radenović, S, Rakočević, V: A note on the equivalence of some metric and cone metric fixed point results. Appl. Math. Lett. 24, 370-374 (2011). doi:10.1016/j.aml.2010.10.030

38. Arandelović, ID, Kečkić, DJ: On nonlinear quasi-contractions on TVS-cone metric spaces. Appl. Math. Lett. 24, 1209-1213 (2011). doi:10.1016/j.aml.2011.02.010

39. Simić, S: A note on Stone's, Baire's, Ky Fan's and Dugundji's theorem in tvs-cone metric spaces. Appl. Math. Lett. 24 999-1002 (2011). doi:10.1016/j.aml.2011.01.014

40. Çakalli, H, Sönmez, A, Genç, C: Metrizability of topological vector space valued cone metric spaces (2010). arXiv:1102.1419

41. Asadi, $M$, Rhoades, BE, Soleimani, H: Some notes on the paper 'The equivalence of cone metric spaces and metric spaces'. Fixed Point Theory Appl. 2012, Article ID 87 (2012). doi:10.1186/1687-1812-2012-87

42. Kadelburg, Z, Radenović, S, Rakočević, V: Remarks on 'Quasi-contraction on a cone metric space'. Appl. Math. Lett. 22, 1674-1679 (2009). doi:10.1016/j.aml.2009.06.003

43. Abdeljawad, T, Rezapour, S: On some topological concepts of TVS-cone metric spaces and fixed point remarks (2011). arXiv:1102.1419

44. Ortega, JM, Rheinboldt, WC: Iterative Solution of Nonlinear Equations in Several Variables. Academic Press, New York (1970)

45. Dudley, RM: On sequential convergence. Trans. Am. Math. Soc. 112, 483-507 (1964)

46. Frič, R: History of sequential convergence spaces. In: Handbook of the History of General Topology, vol. 1, pp. 343-355. Kluwer Academic, Dordrecht (1997)

47. Rus, IA: Picard operators and applications. Sci. Math. Jpn. 58, 191-219 (2003)

48. Petruşel, A, Rus, IA: Fixed point theorems in ordered L-spaces. Proc. Am. Math. Soc. 134, 411-418 (2006). doi:10.1090/50002-9939-05-07982-7

49. Gutierres, G, Hofmann, D: Axioms for sequential convergence. Appl. Categ. Struct. 15, 599-614 (2007). doi:10.1007/s10485-007-9095-2

50. Jakubík, J: On convergence in linear spaces. Mat.-Fyz. čas. 6, 57-67 (1956) (in Slovak)

51. De Pascale, E, Marino, G, Pietramala, P: The use of the $E$-metric spaces in the search for fixed points. Matematiche 48 , 367-376 (1993)

52. Franklin, SP: Spaces in which sequences suffice. Fundam. Math. 57, 107-115 (1965)

53. Goreham, A: Sequential convergence in topological spaces (2004). arXiv:math/0412558

54. Krein, MG, Rutman, MA: Linear operators leaving invariant a cone in a Banach space. Usp. Mat. Nauk 3(1), 3-95 (1948)

55. Krasnoselskii, MA: Positive Solutions of Operator Equations. Gosudarstv. Izdat. Fiz. Mat. Lit., Moscow (1962) (in Russian)

56. Deimling, K: Nonlinear Functional Analysis. Springer, Berlin (1985)

57. Zeidler, E: Applied Functional Analysis: Applications to Mathematical Physics. Applied Mathematical Sciences, vol. 108. Springer, New York (1995)

58. Aliprantis, CD, Tourky, R: Cones and Duality. Graduate Studies in Mathematics, vol. 84. Am. Math. Soc., Providence (2007)

59. Rudin, W: Functional Analysis. McGraw-Hill, New York (1973)

60. Vandergraft, JS: Newton's method for convex operators in partially ordered spaces. SIAM J. Numer. Anal. 4, 406-432 (1967)

61. Kantorovich, LV: Sur la théorie générate des opérations dans les espaces semi-ordonnés. C. R. (Dokl.) Acad. Sci. URSS 1(10), 213-286 (1936)

62. Perov, Al: On the Cauchy problem for a system of ordinary differential equations. In: Approximate Methods of Solving Differential Equations, vol. 2, pp. 115-134. Naukova Dumka, Kiev (1964) (in Russian)

63. Katok, S: p-Adic Analysis in Comparison with Real. Am. Math. Soc., Providence (2003)

64. Engler, AJ, Prestel, A: Valued Fields. Springer Monographs in Mathematics. Springer, Berlin (2005)

65. Feng, Y, Mao, W: The equivalence of cone metric spaces and metric spaces. Fixed Point Theory 11, 259-263 (2010)

66. Rheinboldt, WC: A unified convergence theory for a class of iterative processes. SIAM J. Numer. Anal. 5, $42-63$ (1968)

67. Gel'man, AE: A certain fixed point principle. Sov. Math. Dokl. 12, 813-816 (1971)

68. Rus, IA: Some fixed point theorems in metric spaces. Rend. Ist. Mat. Univ. Trieste 3, 169-172 (1971)

69. Rus, IA: Generalized Contractions and Applications. Cluj University Press, Cluj-Napoca (2001)

70. Kasahara, S: Some fixed point and coincidence theorems in L-spaces. Kobe J. Math. 3, 181-188 (1975)

71. Hicks, TL, Rhoades, BE: A Banach type fixed point theorem. Math. Jpn. 24, 327-330 (1980)

72. Park, S: A unified approach to fixed points of contractive maps. J. Korean Math. Soc. 16, 95-105 (1980)

73. Proinov, PD: A generalization of the Banach contraction principle with high order of convergence of successive approximations. Nonlinear Anal. 67, 38-62 (2007). doi:10.1016/j.na.2006.09.008

74. Proinov, PD: New general convergence theory for iterative processes and its applications to Newton-Kantorovich type theorems. J. Complex. 26, 3-42 (2010). doi:10.1016/j.jco.2009.05.001

75. Banach, S: Sur les operations dans les ensembles abstraits et leurs applications aux equations integrals. Fundam. Math. 3, 133-181 (1922)

76. Kirk, WA: Fixed Point Theory: A Brief Survey. Universidad de Los Andes, Merida (1990)

77. Berinde, V: Iterative Approximation of Fixed Points. Lecture Notes in Mathematics, vol. 1912. Springer, Berlin (2007)

78. Proinov, PD: General local convergence theory for a class of iterative processes and its applications to Newton's method. J. Complex. 25, 38-62 (2009). doi:10.1016/j.jco.2008.05.006 
79. Sendov, B, Andreev, A, Kjurkchiev, N: Numerical solution of polynomial equations. In: Handbook of Numerical Analysis, vol. III, pp. 625-778. Elsevier, Amsterdam (1994). doi:10.1016/S1570-8659(05)80019-5

80. Petković, M: Point Estimation of Root Finding Methods. Lecture Notes in Mathematics, vol. 1933. Springer, Berlin (2008). doi:10.1007/978-3-540-77851-6

doi:10.1186/1687-1812-2013-103

Cite this article as: Proinov: A unified theory of cone metric spaces and its applications to the fixed point theory. Fixed Point Theory and Applications 2013 2013:103.

Submit your manuscript to a SpringerOpen ${ }^{\circ}$ journal and benefit from:

- Convenient online submission

- Rigorous peer review

- Immediate publication on acceptance

Open access: articles freely available online

- High visibility within the field

- Retaining the copyright to your article 